\title{
On Complex-valued 2D Eikonals. Part Four: Continuation Past a Caustic
}

\author{
Rolando Magnanini and Giorgio Talenti
}

\begin{abstract}
Theories of monochromatic high-frequency electromagnetic fields have been designed by Felsen, Kravtsov, Ludwig and others with a view to portraying features that are ignored by geometrical optics. These theories have recourse to eikonals that encode information on both phase and amplitude - in other words, are complex-valued. The following mathematical principle is ultimately behind the scenes: any geometric optical eikonal, which conventional rays engender in some light region, can be consistently continued in the shadow region beyond the relevant caustic, provided an alternative eikonal, endowed with a non-zero imaginary part, comes on stage.

In the present paper we explore such a principle in dimension 2 . We investigate a partial differential system that governs the real and the imaginary parts of complex-valued two-dimensional eikonals, and an initial value problem germane to it. In physical terms, the problem in hand amounts to detecting waves that rise beside, but on the dark side of, a given caustic. In mathematical terms, such a problem shows two main peculiarities: on the one hand, degeneracy near the initial curve; on the other hand, ill-posedness in the sense of Hadamard. We benefit from using a number of technical devices: hodograph transforms, artificial viscosity, and a suitable discretization. Approximate differentiation and a parody of the quasi-reversibility method are also involved. We offer an algorithm that restrains instability and produces effective approximate solutions.
\end{abstract}

Mathematics Subject Classification (2000). Primary 35F25, 35Q60, 78A05; Secondary 65D25.

Keywords. Eikonal, caustics, evanescent waves, nonlinear partial differential systems, initial value problems, hodograph transforms, viscosity, quasi-reversibility, approximate differentiation. 


\section{R. Magnanini and G. Talenti}

\section{Introduction}

1.1. Geometrical optics fits well a variety of issues, but especially survives as an asymptotic theory of monochromatic high-frequency electromagnetic fields — [14], [56], [76], [80], [98], [99], [100], [105] and [106], [116], [137], [145], [184] are selected apropos references. Generalizations have been worked out by Felsen, Kravtsov, Ludwig and their followers - see e.g. [39] and [40], [60], [71] and [72], [87], [112],[113], [114] and [115], [131], [135] and [136], or consult [26], [37], [117]. One is enough for successfully modeling basic optical processes, such as the propagation of light and the development of caustics. The others embrace geometrical optics and are additionally apt to account for certain optical phenomena - for instance, the rise of evanescent waves past a caustic - that are beyond the reach of geometrical optics. A leitmotif of these is allowing a keynote parameter to adjust itself to a standard equation, and simultaneously take complex values.

The partial differential equation

$$
\left(\frac{\partial w}{\partial x}\right)^{2}+\left(\frac{\partial w}{\partial y}\right)^{2}=n^{2}(x, y)
$$

underlies the mentioned theories in case the spacial dimension is 2. Here $x$ and $y$ denote rectangular coordinates in the Euclidean plane; $n$ is a realvalued, strictly positive function of $x$ and $y ; w$ is allowed to take both real and complex values. Function $n$ represents the refractive index of an appropriate (isotropic, non-conducting) two-dimensional medium - its reciprocal stands for velocity of propagation. Function $w$ is named eikonal according to usage, and relates to the asymptotic behavior of an electromagnetic field as the wave number grows large - the real part of $w$ accounts for oscillations, the imaginary part of $w$ accounts for damping. Throughout the present paper we assume the refractive index is conveniently smooth, and consider sufficiently smooth eikonals.

1.2. Geometrical optics deals exclusively with real-valued eikonals, by definition. The partial differential system

$$
\begin{aligned}
u_{x}^{2}+u_{y}^{2}-v_{x}^{2}-v_{y}^{2} & =n^{2}(x, y) \\
u_{x} v_{x}+u_{y} v_{y} & =0
\end{aligned}
$$

governs complex-valued eikonals, i.e. those solutions to (1.1) that obey

$$
u=\operatorname{Re} w, \quad v=\operatorname{Im} w .
$$

Observe the architecture of (1.2): gradients are involved through their orthogonal invariants - lengths and inner product - only. Observe also the 
following properties, which result from a standard test and easy algebraic manipulations. First, system (1.2) is elliptic-parabolic or degenerate elliptic. Second, a solution array $[u v]$ to (1.2) is elliptic if and only if its latter entry $v$ is free from critical points.

The Bäcklund transformation, which relates $u$ and $v$ through

$$
\begin{gathered}
\nabla v=f\left[\begin{array}{cc}
0 & -1 \\
1 & 0
\end{array}\right] \nabla u, \\
f^{2}=1-\frac{n^{2}}{|\nabla u|^{2}}, \quad \operatorname{sgn} f=\operatorname{sgn}\left(u_{x} v_{y}-u_{y} v_{x}\right),
\end{gathered}
$$

and implies both

$$
|\nabla u| \geq n
$$

and

$$
\operatorname{div}\left\{\sqrt{1-\frac{n^{2}}{|\nabla u|^{2}}} \nabla u\right\}=0,
$$

is another, decoupled form of (1.2).

System (1.2) discloses two scenarios - the former is tantamount to conventional geometrical optics, the latter opens up new vistas. Either the equations

$$
u_{x}^{2}+u_{y}^{2}=n^{2} \text { and } v_{x}=v_{y}=0
$$

hold, or the inequalities

$$
|\nabla u|>n \text { and }|\nabla v|>0,
$$

and the following equations prevail.

$$
\begin{gathered}
\nabla u=\frac{1}{f}\left[\begin{array}{cc}
0 & 1 \\
-1 & 0
\end{array}\right] \nabla v, \quad \frac{1}{f^{2}}=1+\frac{n^{2}}{|\nabla v|^{2}} \\
\operatorname{div}\left\{\sqrt{1+\frac{n^{2}}{|\nabla v|^{2}}} \nabla v\right\}=0 \\
\left(|\nabla u|^{4}-n^{2} u_{y}^{2}\right) u_{x x}+2 n^{2} u_{x} u_{y} u_{x y}+\left(|\nabla u|^{4}-n^{2} u_{x}^{2}\right) u_{y y}=n|\nabla u|^{2}\langle\nabla n, \nabla u\rangle, \\
\left(|\nabla v|^{4}+n^{2} v_{y}^{2}\right) v_{x x}-2 n^{2} v_{x} v_{y} v_{x y}+\left(|\nabla v|^{4}+n^{2} v_{x}^{2}\right) v_{y y}+n|\nabla v|^{2}\langle\nabla n, \nabla v\rangle=0 .
\end{gathered}
$$

Equations (1.5) represent another Bäcklund transformation, which inverts the previous one and imply (1.6). Quasi-linear partial differential equations of the second order in non-divergence form appear in (1.7) and (1.8). The former has a mixed elliptic-hyperbolic character: a solution $u$ is elliptic or hyperbolic depending on whether the length of $\nabla u$ exceeds, or 


\section{R. Magnanini and G. Talenti}

is smaller than $n$. The latter is elliptic-parabolic or degenerate elliptic: a solution $v$ such that $\nabla v$ is free from zeros is elliptic, degeneracy occurs at the critical points of $v$.

Any sufficiently smooth solution to (1.4) satisfies (1.7). An elliptic solution to (1.7) coincides with the former entry of an elliptic solution to (1.2). A real-valued function $u$, smooth and without critical points, is a hyperbolic solution to (1.7) if and only if two real-valued smooth, essentially distinct eikonals $\varphi$ and $\psi$ exist such that $u$ is the average of $\varphi$ and $\psi$, i.e.

$$
\varphi_{x}^{2}+\varphi_{y}^{2}=n^{2}, \quad \psi_{x}^{2}+\psi_{y}^{2}=n^{2}, \quad \varphi_{x} \psi_{y}-\varphi_{y} \psi_{x} \neq 0
$$

and

$$
u=\frac{1}{2}(\varphi+\psi)
$$

Any sufficiently smooth solution to (1.6) satisfies (1.8). Any elliptic solution to (1.8) satisfies (1.6). However, a solution to (1.8) need not satisfy (1.6): for instance, perfectly smooth solutions to (1.8) exist whose gradient vanishes exclusively in a set of measure 0 , and which make the left-hand side of (1.6) a non-zero distribution.

A variational approach to equations (1.6) and (1.8) can be summarized thus. Let $J$ be endowed with an appropriate domain and obey

$$
J(v)=\iint j\left(\frac{|\nabla v|}{n}\right) n^{2} d x d y
$$

for any $v$ from that domain - here $j$ is the arc length along a parabola, videlicet

$$
j(\rho)=\frac{\rho}{2} \sqrt{1+\rho^{2}}+\frac{1}{2} \log \left(\rho+\sqrt{1+\rho^{2}}\right)
$$

for any real $\rho$. The following properties hold. (i) $J$ is convex, coercive and sub-differentiable, but not Fréchet-differentiable. (ii) Any critical point of $J$, i.e. any function $v$ such that

$$
\partial J(v) \ni 0,
$$

satisfies (1.6) in any open set $\mathcal{O}$ such that

$\mathcal{O}$ is essentially contained in $\{(x, y) \in$ domain of $v: \nabla v(x, y) \neq 0\}$.

Consequently, a critical point of $J$ solves a free-boundary problem for equation (1.6) — the relevant free boundary is

$$
\text { (domain of } v) \cap \partial\{(x, y) \in \text { domain of } v: \nabla v(x, y) \neq 0\}
$$

and plays the role of a caustic. (iii) Any function $v$ such that

$$
J(v)=\text { minimum }
$$


satisfies (1.8) in an appropriate viscosity sense. In other words, a minimizer of $J$ solves in a generalized sense a boundary value problem for equation (1.8).

An early treatment of (1.2) traces back to [61]. Further apropos information is offered in [138], [139], [140], and [141], where solutions in closed form, qualitative features, exterior boundary value problems, related free boundaries, variational and viscosity methods are discussed.

1.3. Geometrical optics ultimately amounts to manipulating: (i) the Riemannian metric known as travel time, videlicet

$$
n(x, y) \sqrt{d x^{2}+d y^{2}}
$$

(ii) appropriate one-parameter families of travel time geodesics — whose members are nicknamed rays; (iii) the envelopes of rays - called caustics.

Geometric optical eikonals are entirely controlled by rays. They shine in light regions (those spanned by relevant rays), burn out beside caustics (where the ray system breaks down), and shut down in shadow regions (that rays avoid). As a consequence, geometrical optics is unable to account for any optical process that takes place beyond a caustic, on the dark side of it. Essentials of two-dimensional geometrical optics (which are well-established but instrumental here) are outlined in an appendix for reader's convenience.

Complex-valued eikonals prove more flexible. The cited work of Felsen, Kravtsov and Ludwig comprehends the following manifesto among other things: complex-valued eikonals are apt to consistently continue geometric optical cognates into shadow regions.

Such a continuation is the main theme of the present paper.

\section{Heuristics}

Let us pave the way by heuristically considering the case where refractive index $n$ is 1 . A classical recipe informs how general geometric optical eikonals can be cooked up: start from a complete integral, derive a one-parameter family of solutions, take the relevant envelope, and shake well. Let $f$ be an arbitrary, but sufficiently smooth, real function. The pair

$$
w=x \cos t+y \sin t+f(t), \quad 0=-x \sin t+y \cos t+f^{\prime}(t)
$$

causes $w$ and $t$ to enjoy the following properties:

$$
\left(\frac{\partial w}{\partial x}\right)^{2}+\left(\frac{\partial w}{\partial y}\right)^{2}=1
$$




\section{R. Magnanini and G. Talenti}

the pertinent eikonal equation governing w;

$$
\cos t \frac{\partial t}{\partial x}+\sin t \frac{\partial t}{\partial y}=0
$$

a Burgers-type equation governing $t$;

$$
w_{x} t_{x}+w_{y} t_{y}=0
$$

showing that the gradients of $w$ and $t$ are orthogonal;

$$
w_{x}=\cos t, \quad w_{y}=\sin t,
$$

a Bäcklund transformation further relating $w$ and $t$. Both the rays of $w$ and the level lines of $t$ are the straight-lines where

$$
-x \sin t+y \cos t+f^{\prime}(t)=0
$$

and $t$ equals a constant. Such straight-lines span the light region and envelope the caustic. We have

$$
-f^{\prime}=\text { the support function of the caustic, }
$$

and

$$
x=f^{\prime \prime}(t) \cos t+f^{\prime}(t) \sin t, \quad y=f^{\prime \prime}(t) \sin t-f^{\prime}(t) \cos t, \quad w=f(t)+f^{\prime \prime}(t)
$$

along the caustic. Therefore the second-order derivatives of $w$ and the gradient of $t$ simultaneously blow up there - in particular, the caustic of $w$ is also the shock-line of $t$.

We claim that both $w$ and $t$ can be continued beyond the caustic, in a subset of the shadow region, if complex values are allowed. Suppose $f$ can be continued by a holomorphic function of a complex variable. (Information on analytic continuation is in the next section.) Let $i=\sqrt{-1}$, the unit imaginary number. Let $u, v, \lambda, \mu$ be real; put the equations

$$
w=u+i v, \quad t=\lambda+i \mu,
$$

and equations (2.1) together, but force (2.1) to produce real $x$ and $y$. The formulas

$$
\begin{aligned}
& x=\frac{\sin \lambda}{\cosh \mu} \operatorname{Re} f^{\prime}(t)+\frac{\cos \lambda}{\sinh \mu} \operatorname{Im} f^{\prime}(t), \\
& y=-\frac{\cos \lambda}{\cosh \mu} \operatorname{Re} f^{\prime}(t)+\frac{\sin \lambda}{\sinh \mu} \operatorname{Im} f^{\prime}(t), \\
& u=\operatorname{Re} f(t)+(x \cos \lambda+y \sin \lambda) \cosh \mu, \\
& v=\operatorname{Im} f(t)+(-x \sin \lambda+y \cos \lambda) \sinh \mu,
\end{aligned}
$$

ensue, then an inspection testifies that the claimed continuation ensues too. 
Incidentally, we have also shown that solutions to the non-viscous Burgers equation can be continued past the shock-line by suitable complexvalued solutions to the same equation.

Note the following. In case

$$
f(t)=t
$$

for every real or complex $t$, the caustic is the unit circle, the rays are halflines tangent to it, the shadow region is the unit disk. Formulas (2.1) and (2.2) become transparent when recast as shown below. Let rectangular and polar coordinates be related by

$$
x=\rho \sin \varphi, y=-\rho \cos \varphi \quad(0 \leq \rho<\infty,-\pi<\varphi \leq \pi) .
$$

A geometrical optical eikonal happens to satisfy

$$
w=\varphi \pm\left\{\arctan \sqrt{\rho^{2}-1}-\sqrt{\rho^{2}-1}\right\}
$$

in the region where

$$
1 \leq \rho<\infty ;
$$

the same eikonal is ipso facto continued by

$$
w=u+i v, u=\varphi, v= \pm\left\{\log \left(\frac{1}{\rho}+\sqrt{\frac{1}{\rho^{2}}-1}\right)-\sqrt{1-\rho^{2}}\right\}
$$

in the region where

$$
0<\rho<1
$$

- $u$ is a helicoid, $v$ is a pseudosphere.

A more exhaustive analysis is carried out in the next section.

\section{Analytic continuation}

3.1. Here we sketch a special method of continuing a two-dimensional geometric optical eikonal past a caustic. Though rigorous, such a method is slightly reminiscent of the so-called theory of complex rays — cf. [37] or [115], for instance. It applies in the case where the refractive index equals 1 , and can be used for simultaneously continuing solutions to non-viscous Burgers equation beyond shock lines. Let us mention that complex-valued solutions to viscous and non-viscous Burgers equation are dealt with in [102], [174] and [101]. 


\section{R. Magnanini and G. Talenti}

3.2. The present method involves analytic continuation from the real-number axis into the complex plane - an ill-posed process in the sense of Hadamard. Let $h$ be a real or complex-valued function of a real variable, or even a list of samples. An analytic continuation of $h$ is a holomorphic function of a complex variable, whose domain surrounds the real axis and whose restriction to the real axis fits $h$ well - in other words, a solution $H$ of the following initial value problem for Cauchy-Riemann equation

$$
\frac{\partial H}{\partial y}=i \frac{\partial H}{\partial x}, \quad H(\cdot, 0) \simeq h .
$$

If $h$ is an analytic function, and is not polluted by noise, an analytic continuation $H$ of $h$ results from obvious formulas. For example,

$$
H(x, y)=\sum_{k=0}^{\infty} \frac{d^{k} h(x)}{d x^{k}} \frac{(i y)^{k}}{k !}
$$

or

$$
H(x, y)=\frac{1}{2 \pi} \int_{-\infty}^{\infty} \exp [i \xi(x+i y)] \widehat{h}(\xi) d \xi
$$

where hat denotes Fourier transformation.

If $h$ collects gross data, an effective analytic continuation $H$ of $h$ can be obtained by analytically continuing an appropriate, smoothed and denoised version of $h$. Consider for instance the case where

$$
\begin{gathered}
-\infty<a<b<\infty, \\
N=\text { an integer larger than } 1,
\end{gathered}
$$

a mesh size is given by

$$
\Delta x=(b-a) /(N-1),
$$

nodes are given by

$$
x_{j}=a+(j-1) \Delta x \quad(j=1, \ldots, N) ;
$$

and $h$ stands for

$$
h_{1}, \ldots, h_{N}
$$

a sequence of noisy samples. 
An ad hoc analytic continuation $H$ solves the following least square problem

$$
\Delta x \sum_{j=1}^{N}\left[H\left(x_{j}, 0\right)-h_{j}\right]^{2}+\frac{\lambda}{2 L} \int_{a-\Delta x / 2}^{b+\Delta x / 2} d x \int_{-L}^{L}|H(x, y)|^{2} d y=\text { minimum }
$$

in a convenient class of holomorphic functions - e.g. the class of trigonometric polynomials of a suitable degree. Here

$$
\frac{\lambda}{2 L} \int_{a-\Delta x / 2}^{b+\Delta x / 2} d x \int_{-L}^{L}|H(x, y)|^{2} d y
$$

plays the role of a penalty; $\lambda$ and $L$ are regulating parameters $-\lambda$ is related to noise, $L$ is related to a priori information.

An explicit expression of $H$ can be derived via discrete Fourier transforms. Suppose for simplicity that $N$ is odd, say

$$
N=2 n+1 \quad(n=1,2, \ldots)
$$

let

$$
T=N \Delta x,
$$

and let DFT be the discrete Fourier transform that obeys the equations

$$
\begin{gathered}
D F T_{k}(h)=\sum_{j=1}^{N} h_{j} \exp \left(-2 \pi i k \frac{x_{j}}{T}\right) \quad(k=-n, \ldots, n), \\
h_{j}=\frac{1}{N} \sum_{k=-n}^{n} D F T_{k}(h) \exp \left(2 \pi i k \frac{x_{j}}{T}\right) \quad(j=1, \ldots, N), \\
\frac{1}{N} \sum_{k=-n}^{n}\left|D F T_{k}(h)\right|^{2}=\sum_{j=1}^{N}\left|h_{j}\right|^{2}
\end{gathered}
$$

— cf. [31], for instance. If

$$
C_{0}=1, C_{k}=\frac{\sinh (4 \pi k L / T)}{4 \pi k L / T}, C_{-k}=C_{k}(k=1, \ldots, n),
$$

then

$$
H(x, y)=\sum_{k=-n}^{n}\left(1+\lambda C_{k}\right)^{-1} D F T_{k}(h) \exp \left(2 \pi i k \frac{x+i y}{T}\right) .
$$




\section{R. Magnanini and G. Talenti}

This is a $T$-periodic trigonometric polynomial of degree $n$ that enjoys the properties

$$
\begin{gathered}
\sum_{j=1}^{N}\left|H\left(x_{j}, 0\right)-h_{j}\right|^{2} \leq\left(\frac{\lambda}{\lambda+1 / C_{n}}\right)^{2} \sum_{j=1}^{N}\left|h_{j}\right|^{2}, \\
\frac{1}{2 L} \int_{a-\Delta x / 2}^{b+\Delta x / 2} d x \int_{-L}^{L}|H(x, y)|^{2} d y \leq \frac{\Delta x}{4 \lambda},
\end{gathered}
$$

and

$$
H(x, y)=\sum_{j=1}^{N} H\left(x_{j}, 0\right) D_{N}\left(2 \pi \frac{x-x_{j}+i y}{T}\right)
$$

- here $D_{N}$ denotes the Dirichlet, or periodic sinc function obeying

$$
D_{N}(x)=\frac{\sin (N x / 2)}{N \sin (x / 2)}
$$

if $x /(2 \pi)$ is not an integer.

More information on analytic continuation can be found in [1], [4], [23], [25], [35], [41]-[42], [50], [54]-[55], [69], [70], [74], [75], [81], [88], [124], [125], $[130],[126],[149]-[150],[152],[187],[192],[194],[211],[215],[221],[223]$, $[226]$.

3.3. Consider a plane curve $C$ that either is inherently smooth or results from a suitable smoothing process of raw data. Assume $C$ is analytic and its curvature vanishes nowhere. For simplicity, assume $C$ is the graph of the equation

$$
y=f(x),
$$

and $f$ is convex.

Alternative parametric representations of $C$, which are instrumental throughout, include

$$
x=t, \quad y=f(t),
$$

where parameter $t$ coincides with the abscissa; and

$$
x=g^{\prime}(t), \quad y=t g^{\prime}(t)-g(t),
$$

where parameter $t$ is the slope of the tangent straight-line. Here $g$ denotes the Legendre conjugate of $f$ - recall from e.g. [190] that $f$ and $g$ are related by

$$
t=f^{\prime}(x), x=g^{\prime}(t), t x=f(x)+g(t), 1=f^{\prime \prime}(x) g^{\prime \prime}(t) .
$$


Curve $C$ changes into a caustic under the following modus operandi. Let

$$
x=\alpha(t), \quad y=\beta(t)
$$

be any parametric representation of $C$, where $\alpha$ and $\beta$ are analytic. Let $\gamma$ and $\kappa$ stand for arc length and curvature, respectively - in other words,

$$
\begin{gathered}
\gamma(t)=\int \sqrt{\alpha^{\prime}(t)^{2}+\beta^{\prime}(t)^{2}} d t, \\
\kappa=\frac{\alpha^{\prime} \beta^{\prime \prime}-\alpha^{\prime \prime} \beta^{\prime}}{\left[\left(\alpha^{\prime}\right)^{2}+\left(\beta^{\prime}\right)^{2}\right]^{3 / 2}} .
\end{gathered}
$$

The pair

$$
\left[\begin{array}{l}
x \\
y
\end{array}\right]=\left[\begin{array}{c}
\alpha(s) \\
\beta(s)
\end{array}\right]+\frac{w-\gamma(s)}{\sqrt{\alpha^{\prime}(s)^{2}+\beta^{\prime}(s)^{2}}}\left[\begin{array}{l}
\alpha^{\prime}(s) \\
\beta^{\prime}(s)
\end{array}\right]
$$

makes $s$ and $w$ curvilinear coordinates. As is easy to see, the level lines of $s$ are tangent straight-lines to $C$, the level lines of $w$ are involutes of $C-$ orthogonal to one another.

Equations (3.5) imply

$$
y \leq f(x),
$$

and

$$
s(x, y)=t, \quad w(x, y)=\gamma(t),
$$

if $x, y$ and $t$ obey (3.4) $-s$ and $w$ live below $C$ and satisfy precise conditions along $C$.

We compute

$$
\frac{\partial(x, y)}{\partial(s, w)}=\left[\begin{array}{cc}
-\beta^{\prime}(s) & \alpha^{\prime}(s) \\
\alpha^{\prime}(s) & \beta^{\prime}(s)
\end{array}\right]\left[\begin{array}{cc}
\kappa(s)[w-\gamma(s)] & 0 \\
0 & {\left[\left(\alpha^{\prime}\right)^{2}+\left(\beta^{\prime}\right)^{2}\right]^{-1 / 2}}
\end{array}\right]
$$

to draw the following set:

$$
\begin{gathered}
\frac{\partial}{\partial x} \alpha(s)+\frac{\partial}{\partial y} \beta(s)=0 \\
\left(\frac{\partial w}{\partial x}\right)^{2}+\left(\frac{\partial w}{\partial y}\right)^{2}=1, \\
\frac{\partial s}{\partial x} \frac{\partial w}{\partial x}+\frac{\partial s}{\partial y} \frac{\partial w}{\partial y}=0 \\
\nabla w=\left[\alpha^{\prime}(s)^{2}+\beta^{\prime}(s)^{2}\right]^{-1 / 2}\left[\begin{array}{c}
\alpha^{\prime}(s) \\
\beta^{\prime}(s)
\end{array}\right], \\
|\nabla s|^{-2}=[w-\gamma(s)]^{2} \kappa(s)^{2}\left[\alpha^{\prime}(s)^{2}+\beta^{\prime}(s)^{2}\right],
\end{gathered}
$$




$$
\left[\begin{array}{cc}
w_{x x} & w_{x y} \\
w_{x y} & w_{y}
\end{array}\right]=\frac{1}{\gamma(s)-w}\left[\begin{array}{c}
-w_{y} \\
w_{x}
\end{array}\right]\left[\begin{array}{ll}
-w_{y} & \left.w_{x}\right]
\end{array}\right.
$$

Equation (3.6) is a conservation law; it reads

$$
\frac{\partial s}{\partial x}+s \frac{\partial s}{\partial y}=0
$$

the standard Burgers equation, if (3.3) is in force. Equation (3.7) is the equation of geometrical optics in hand. Equation (3.8) shows that the gradients of $s$ and $w$ are orthogonal. Equation (3.9) shows that both $C$ and the tangent straight-lines to $C$ are lines of steepest descent of $w$; it also shows that the straight-lines in question are isoclines of $w$. Equation (3.9) can be viewed as a Bäcklund transformation, which converts any solution to (3.6) into a solution to (3.7). It reads

$$
\nabla w=\left[1+f^{\prime}(s)^{2}\right]^{-1 / 2}\left[\begin{array}{c}
1 \\
f^{\prime}(s)
\end{array}\right], \quad s=g^{\prime}\left(\frac{w_{y}}{w_{x}}\right),
$$

or simply

$$
\nabla w=\left[1+s^{2}\right]^{-1 / 2}\left[\begin{array}{l}
1 \\
s
\end{array}\right], \quad s=\frac{w_{y}}{w_{x}},
$$

depending on whether (3.2) or (3.3) is in effect. Equations (3.10) and (3.11) show that both the gradient of $s$ and the second-order derivatives of $w$ blow up near $C$.

We infer that $s$ is governed by a Burgers-type equation, and develops shocks along $C$. The following objects $-w, C$, the tangent straight-lines to $C$, and the region below $C$ - are a geometric optical eikonal, the relevant caustic, the rays, and the light region, respectively.

We now claim: (i) $s$ and $w$ can be continuously extended into the region where

$$
y>f(x)
$$

the dark side of $C$, if suitable imaginary parts are provided; (ii) the relevant extensions obey equations (3.6) to (3.11).

The points above $C$ are reached by no tangent straight-line to $C$, of course. We insist in drawing tangent straight-lines from these points, but allow complex slopes. In other words, we recast (3.5) this way

$$
\begin{gathered}
-\beta^{\prime}(s)[x-\alpha(s)]+\alpha^{\prime}(s)[y-\beta(s)]=0, \\
{[w-\gamma(s)]\left[\alpha^{\prime}(s)^{2}+\beta^{\prime}(s)^{2}\right]^{1 / 2}=\alpha^{\prime}(s)[x-\alpha(s)]+\beta^{\prime}(s)[y-\beta(s)],}
\end{gathered}
$$

and force such equations to hold in the situation where

$$
\operatorname{Re}(s)=\lambda, \quad \operatorname{Im}(s)=\mu, \quad \operatorname{Im}(x)=\operatorname{Im}(y)=0 .
$$


The following formulas result:

$$
\begin{aligned}
& x=\frac{\operatorname{Im}\left[\overline{\alpha^{\prime}(s)}\left(\alpha(s) \beta^{\prime}(s)-\alpha^{\prime}(s) \beta(s)\right)\right]}{\operatorname{Im}\left[\overline{\alpha^{\prime}(s)} \beta^{\prime}(s)\right]}, \\
& y=\frac{\operatorname{Im}\left[\overline{\beta^{\prime}(s)}\left(\beta(s) \alpha^{\prime}(s)-\beta^{\prime}(s) \alpha(s)\right)\right]}{\operatorname{Im}\left[\overline{\beta^{\prime}(s)} \alpha^{\prime}(s)\right]}, \\
& s=\lambda+i \mu, \\
& w=\gamma(s)-\frac{1}{\sqrt{\alpha^{\prime}(s)^{2}+\beta^{\prime}(s)^{2}}}\left\{\overline{\alpha^{\prime}(s)} \frac{\operatorname{Im} \beta(s)}{\operatorname{Im}\left[\overline{\alpha^{\prime}(s)} \beta^{\prime}(s)\right]}+\overline{\beta^{\prime}(s)} \frac{\operatorname{Im} \alpha(s)}{\operatorname{Im}\left[\overline{\beta^{\prime}(s)} \alpha^{\prime}(s)\right]}\right\},
\end{aligned}
$$

where $\alpha, \beta$ and $\gamma$ stand for the analytic continuations of the original objects.

Formulas (3.13) answer the claim. Among other things, they give

$$
\begin{array}{r}
{\left[\begin{array}{l}
x \\
y
\end{array}\right]=\left[\begin{array}{l}
\alpha(\lambda) \\
\beta(\lambda)
\end{array}\right]+\frac{d A(\lambda)}{d \lambda}\left[\begin{array}{l}
\alpha^{\prime}(\lambda) \\
\beta^{\prime}(\lambda)
\end{array}\right] \mu^{2}+B(\lambda)\left[\begin{array}{c}
-\beta^{\prime}(\lambda) \\
\alpha^{\prime}(\lambda)
\end{array}\right] \mu^{2}+O\left(\mu^{4}\right),} \\
A=-\frac{1}{3} \log |\kappa|-\frac{1}{2} \log \sqrt{\left(\alpha^{\prime}\right)^{2}+\left(\beta^{\prime}\right)^{2}}, \quad B=\frac{\kappa}{2} \sqrt{\left(\alpha^{\prime}\right)^{2}+\left(\beta^{\prime}\right)^{2}}
\end{array}
$$

as $\mu$ approaches zero, and

$$
\operatorname{det} \frac{\partial(x, y)}{\partial(\lambda, \mu)}=\frac{\left|\alpha^{\prime}(s) \operatorname{Im} \beta(s)-\beta^{\prime}(s) \operatorname{Im} \alpha(s)\right|^{2}\left|\alpha^{\prime}(s) \beta^{\prime \prime}(s)-\alpha^{\prime \prime}(s) \beta^{\prime}(s)\right|^{2}}{\left[\operatorname{Im} \overline{\alpha^{\prime}(s)} \beta^{\prime}(s)\right]^{3}}
$$

consequently

$$
y-f(x)=\frac{1}{2} f^{\prime \prime}(\alpha(\lambda))[\operatorname{Im} \alpha(\lambda+i \mu)]^{2}+O\left(\mu^{4}\right)
$$

and

$$
\operatorname{det} \frac{\partial(x, y)}{\partial(\lambda, \mu)}=\mu\left[\alpha^{\prime}(\lambda) \beta^{\prime \prime}(\lambda)-\alpha^{\prime \prime}(\lambda) \beta^{\prime}(\lambda)+O\left(\mu^{2}\right)\right]
$$

as $\mu$ approaches zero. Thus (3.13) imply (3.12), as well as

$$
\operatorname{det} \frac{\partial(x, y)}{\partial(\lambda, \mu)} \neq 0
$$

if $\mu$ is different from, and sufficiently close to 0 .

3.4. Here is an example. A catenary is the graph of either the equation

$$
y=\cosh x
$$

or the equations

$$
x=\log \left(t+\sqrt{1+t^{2}}\right), \quad y=\sqrt{1+t^{2}},
$$




\section{R. Magnanini and G. Talenti}

where parameter $t$ coincides with both an arc length and the slope of the tangent straight-line.

Consider solutions to the equations

$$
\left(\frac{\partial w}{\partial x}\right)^{2}+\left(\frac{\partial w}{\partial y}\right)^{2}=1, \quad \frac{\partial s}{\partial x}+s \frac{\partial s}{\partial y}=0
$$

which obey the conditions

$$
w(x, y)=s(x, y)=t
$$

as $x$ and $y$ obey (3.15). Function $w$ and the catenary in question are an eikonal and the relevant caustic, respectively; $s$ obeys Burgers equation, takes a constant value on each tangent straight-line to the catenary and develops shocks along the catenary.

The light region is the set where

$$
-\infty<x<\infty, \quad y \leq \cosh x
$$

the shadow region is the set above the catenary, where

$$
-\infty<x<\infty, \quad \cosh x<y
$$

The pair

$$
\begin{gathered}
s x-y=s \log \left(s+\sqrt{1+s^{2}}\right)-\sqrt{1+s^{2}}, \\
w=\frac{1}{\sqrt{1+s^{2}}}\left[x+s y-\log \left(s+\sqrt{1+s^{2}}\right)\right],
\end{gathered}
$$

makes $s$ and $w$ implicit functions of $x$ and $y$ in the light region. Eikonal $w$ and its partner $s$ can be continued in a subset of the shadow region via the equations

$$
\begin{aligned}
& x=\lambda+\tanh \lambda(\mu \cot \mu-1), \\
& y=\frac{1}{\cosh \lambda}\left(\sinh ^{2} \lambda \frac{\mu}{\sin \mu}+\mu \sin \mu+\cos \mu\right), \\
& s=\sinh (\lambda+i \mu), \\
& w=\sinh \lambda \frac{\mu}{\sin \mu}+\frac{i}{\cosh \lambda}(\sin \mu-\mu \cos \mu)
\end{aligned}
$$

- here $\lambda$ and $\mu$ are parameters such that

$$
-\infty<\lambda<\infty, \quad 0 \leq \mu \leq \pi / 2 .
$$

The foregoing equations imply that

$$
y-\cosh x=(\cosh \lambda) \mu^{2}\left[\frac{1}{2}+O\left(\mu^{2}\right)\right] \text {, }
$$


as $\mu$ approaches 0 . Furthermore,

$$
\begin{gathered}
\operatorname{det} \frac{\partial(x, y)}{\partial(\lambda, \mu)}=\frac{\left[\mu^{2}+\tanh ^{2} \lambda(1-\mu \cot \mu)^{2}\right]\left(\sinh ^{2} \lambda+\cos ^{2} \mu\right)}{\cosh \lambda \sin \mu}, \\
\frac{\partial w}{\partial x}=\frac{1}{\cosh (\lambda+i \mu)}, \frac{\partial w}{\partial y}=\tanh (\lambda+i \mu)
\end{gathered}
$$

- in particular, a singularity occurs at the point whose coordinates are

$$
x=0, \quad y=\pi / 2 .
$$

Figures 1 and 2 show plots of the imaginary parts of $w$ and $s$.

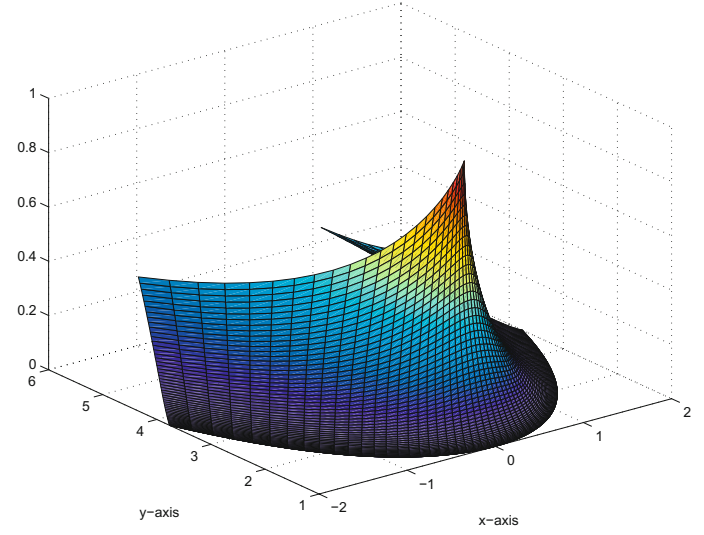

Figure 1. Eikonal equation: the imaginary part of $w$ beyond a caustic.

\section{Main statements}

The most exhaustive method of continuing a two-dimensional geometric optical eikonal beyond a caustic consists perhaps in tackling a certain initial value problem - singular and ill-posed - for system (1.2). Such a problem is described in items (i) and (ii) below, and Figure 3.

(i) An initial curve IC is given.

The following alternative applies: either IC is specified exactly - no error infects the definition of IC; or else IC is a phantom - some coarse and polluted sampling of IC is gotten. In the former case assume IC is smooth enough. In the latter case recover IC, i.e. feed the available data into an 


\section{R. Magnanini and G. Talenti}

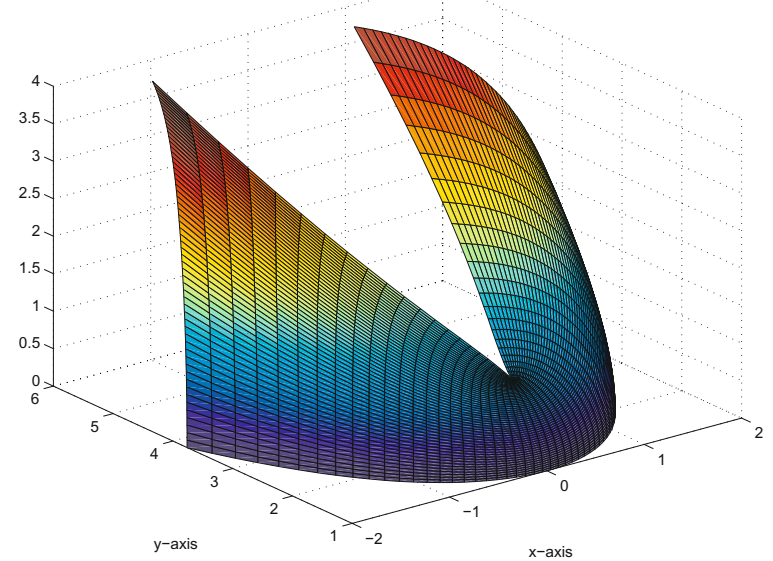

FIgURE 2. Burgers equation: the imaginary part of $s$ beyond a shock-line.

$$
\begin{aligned}
& w=\text { Re } w \\
& w_{x}^{2}+w_{y}^{2}=n^{2}(x, y) \\
& \text { light }
\end{aligned}
$$

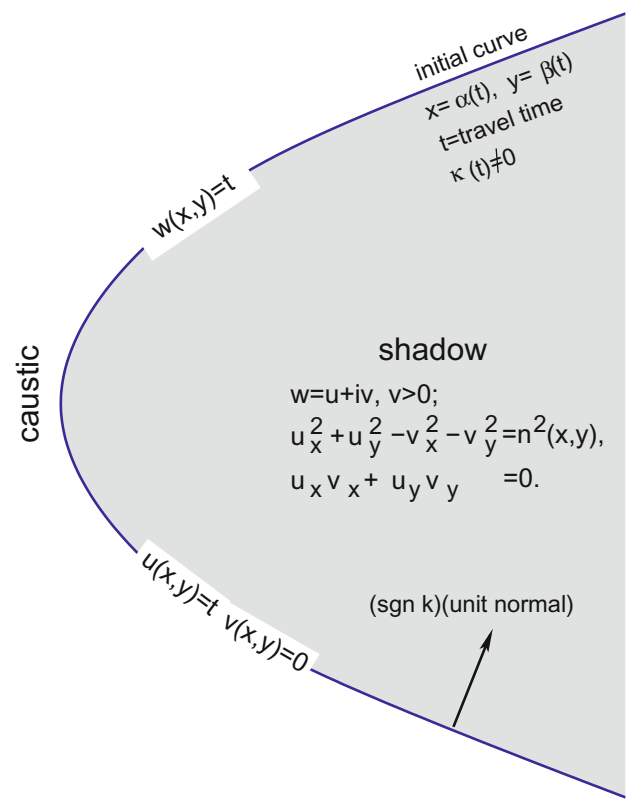

Figure 3. A geometric optical eikonal and its continuation past a caustic. 
appropriate denoising process, and then elect the consequent output as an operative substitute of IC. (Ad hoc tools can be found in Section 7.)

Represent IC (either the authentic one, or else its surrogate) by the equations

$$
x=\alpha(t), \quad y=\beta(t),
$$

and adjust parameter $t$ so as

$$
t=a \text { travel time }
$$

without any loss of generality.

Assume travel time is an extra metric in action and the relevant geodesic curvature of IC is free from zeroes. In other words, postulate that (4.1) and either of the equations

$$
\begin{gathered}
\kappa(\text { velocity })^{3}=\text { Geodesic curvature, } \\
\kappa(\text { velocity })^{2}=\text { Euclidean curvature }-\langle\text { unit normal, } \nabla \log n(x, y)\rangle
\end{gathered}
$$

result in

$$
\kappa \text { vanishes nowhere. }
$$

(ii) A pair $[u v]$ is sought that obeys system (1.2) and fulfills the following conditions. First,

$$
u(x, y)=t, \quad v(x, y)=0,
$$

if $x, y$ and $t$ are subject to (4.1). Second, $u$ and $v$ are defined in the side of IC that

$$
(\operatorname{sgn} \kappa) \times(\text { unit normal to IC })
$$

points to.

Arguments from the appendix allow us to comment as follows. IC and the mentioned side of it can be viewed as a caustic and a shadow region, respectively. Any geometric optical eikonal, which makes IC a caustic, lives in the illuminated side of IC; the complex-valued eikonal, whose real part is $u$ and whose imaginary part is $v$, lives in the opposite, dark side of IC instead. Both the former and the latter equal a travel time along IC. An extension of the geometric optical eikonal in hand ensues. Such an extension does obey the eikonal equation, lives in both the light region and a subset of the shadow region, and takes complex values where shadow prevails. In physical terms, problem (i) and (ii) accepts a caustic in input, then models evanescent waves that rise in the dark side of it.

In the present paper we focus our attention on solutions $[u v]$ to the problem (i) and (ii) that meet the following extra requirements: 


\section{R. Magnanini and G. Talenti}

(iii) they are elliptic;

(iv) their Jacobian determinant obeys

$$
\operatorname{sgn}\left(u_{x} v_{y}-u_{y} v_{x}\right)=\operatorname{sgn} \kappa .
$$

Condition (iii) ensures that the latter entry $v$ is not constant; as will emerge from subsequent developments, initial conditions plus conditions (iii) and (iv) ensure that the same entry is nonnegative.

The solutions to problem (i)-(iv) develop singularities near IC, as any geometric optical eikonal does in the vicinity of the relevant caustic. We will show in Section 6 that they obey the expansions

$$
u(x, y)=s+o(r), v(x, y)=\frac{2 \sqrt{2}}{3}|\kappa(s)|^{1 / 2}|r|^{3 / 2}+o\left(r^{3 / 2}\right)
$$

as $(x, y)$ belongs to the appropriate side of, and is close enough to IC. Here $r$ and $s$ stand for the curvilinear coordinates described in the appendix informally, $r$ is a signed distance from IC, $s$ is a lifting of a travel time inherent to IC. Note the physical meaning of (4.6): the damping effects, which are encoded in the imaginary part of a complex-valued eikonal, are tuned by the geodesic curvature of the relevant caustic.

Problem (i)-(iv) is recast in the next section into a more tractable form.

\section{Framework}

5.1. A convenient coordinate system must be called for. We choose to recast (1.2) by reversing the roles of dependent and independent variables - i.e. we think of $u$ and $v$ as curvilinear coordinates, and think of $x$ and $y$ as functions of $u$ and $v$. In other words, we subject (1.2) to the change of variables that is called hodograph transformation at times — see [64] and [227], for instance.

Let $[u v]$ be any smooth elliptic solution to (1.2), and observe the following.

(i) The level lines of $u$ and those of $v$ are free from singular points, and cross at a right angle. Moreover, the Jacobian determinant

$$
u_{x} v_{y}-u_{y} v_{x} \text { vanishes nowhere. }
$$

Let

$$
[u v] \mapsto[x(u, v) y(u, v)] \text { be a local inverse of }[x y] \mapsto[u(x, y) v(x, y)] \text {, }
$$


then observe the following equation

$$
\left(u_{x} v_{y}-u_{y} v_{x}\right)\left(x_{u} y_{v}-x_{v} y_{u}\right)=1
$$

and the propositions (ii)-(iv) below.

(ii) The following partial differential system holds:

$$
1 / E-1 / G=1, \quad F=0 .
$$

Here

$$
E=n^{2}(x, y)\left(x_{u}^{2}+y_{u}^{2}\right), F=n^{2}(x, y)\left(x_{u} x_{v}+y_{u} y_{v}\right), G=n^{2}(x, y)\left(x_{v}^{2}+y_{v}^{2}\right)
$$

- in other words,

$$
n^{2}(x, y)\left((d x)^{2}+(d y)^{2}\right)=E(d u)^{2}+2 F d u d v+G(d v)^{2} .
$$

(iii) The following systems and equations hold:

$$
\begin{gathered}
\frac{\partial}{\partial u}\left[\begin{array}{l}
x \\
y
\end{array}\right]=f\left[\begin{array}{cc}
0 & 1 \\
-1 & 0
\end{array}\right] \frac{\partial}{\partial v}\left[\begin{array}{l}
x \\
y
\end{array}\right], \\
\frac{\partial}{\partial v}\left[\begin{array}{l}
x \\
y
\end{array}\right]=\frac{1}{f}\left[\begin{array}{cc}
0 & -1 \\
1 & 0
\end{array}\right] \frac{\partial}{\partial u}\left[\begin{array}{l}
x \\
y
\end{array}\right], \\
\operatorname{sgn} f=\operatorname{sgn}\left(x_{u} y_{v}-x_{v} y_{u}\right), \\
\frac{1}{f^{2}}=1+n^{2}(x, y)\left(x_{v}^{2}+y_{v}^{2}\right), \quad f^{2}=1-n^{2}(x, y)\left(x_{u}^{2}+y_{u}^{2}\right) .
\end{gathered}
$$

(iv) The following equations hold:

$$
\begin{gathered}
f^{2}=\frac{1-n^{2}(x, y) x_{u}^{2}}{1+n^{2}(x, y) x_{v}^{2}}, f^{2}=\frac{1-n^{2}(x, y) y_{u}^{2}}{1+n^{2}(x, y) y_{v}^{2}} \\
\frac{\partial^{2}}{\partial u^{2}}\left[\begin{array}{l}
x \\
y
\end{array}\right]+f^{4} \frac{\partial^{2}}{\partial v^{2}}\left[\begin{array}{l}
x \\
y
\end{array}\right]=\left(1-f^{2}\right)\left[\begin{array}{cc}
x_{u}^{2}-y_{u}^{2} & -2 x_{u} y_{u} \\
2 x_{u} y_{u} & x_{u}^{2}-y_{u}^{2}
\end{array}\right] \nabla \log n(x, y)
\end{gathered}
$$

— in the event that $n$ is identically 1 , these equations read

$$
x_{u u}+\left(\frac{1-x_{u}^{2}}{1+x_{v}^{2}}\right)^{2} x_{v v}=0, \quad y_{u u}+\left(\frac{1-y_{u}^{2}}{1+y_{v}^{2}}\right)^{2} y_{v v}=0 .
$$

Proof of (i). System (1.2) tells us that

$$
|\nabla u|>0,
$$

and that the gradients of $u$ and $v$ are orthogonal. Ellipticity gives

$$
|\nabla v|>0 \text {. }
$$


The equation

$$
\left(u_{x} v_{y}-u_{y} v_{x}\right)^{2}=|\nabla u|^{2}|\nabla v|^{2}-\langle\nabla u, \nabla v\rangle^{2}
$$

concludes the proof.

Proof of (ii). Since

$$
\begin{gathered}
\frac{\partial(u, v)}{\partial(x, y)} \times \frac{\partial(x, y)}{\partial(u, v)}=\left[\begin{array}{cc}
1 & 0 \\
0 & 1
\end{array}\right], \\
\frac{\partial(u, v)}{\partial(x, y)} \times\left[\frac{\partial(u, v)}{\partial(x, y)}\right]^{T}=\left[\begin{array}{cc}
|\nabla u|^{2} & \langle\nabla u, \nabla v\rangle \\
\langle\nabla u, \nabla v\rangle & |\nabla v|^{2}
\end{array}\right], \\
{\left[\frac{\partial(x, y)}{\partial(u, v)}\right]^{T} \times \frac{\partial(x, y)}{\partial(u, v)}=\left[\begin{array}{cc}
x_{u}^{2}+y_{u}^{2} & x_{u} x_{v}+y_{u} y_{v} \\
x_{u} x_{v}+y_{u} y_{v} & x_{v}^{2}+y_{v}^{2}
\end{array}\right],}
\end{gathered}
$$

we have

$$
\begin{aligned}
\frac{1}{x_{u}^{2}+y_{u}^{2}}= & |\nabla u|^{2}-\frac{\langle\nabla u, \nabla v\rangle^{2}}{|\nabla v|^{2}}, \frac{1}{x_{v}^{2}+y_{v}^{2}}=|\nabla v|^{2}-\frac{\langle\nabla u, \nabla v\rangle^{2}}{|\nabla u|^{2}}, \\
& x_{u} x_{v}+y_{u} y_{v}=-\langle\nabla u, \nabla v\rangle\left(x_{u} y_{v}-x_{v} y_{u}\right)^{2}
\end{aligned}
$$

The conclusion ensues.

Proof of (iii) and (iv). The latter equation from (5.2) yields

$$
x_{u}: y_{v}=y_{u}:\left(-x_{v}\right),
$$

hence the following systems result:

$$
\left[\begin{array}{l}
x_{u} \\
y_{u}
\end{array}\right]=f\left[\begin{array}{cc}
0 & 1 \\
-1 & 0
\end{array}\right]\left[\begin{array}{l}
x_{v} \\
y_{v}
\end{array}\right], \quad\left[\begin{array}{l}
x_{v} \\
y_{v}
\end{array}\right]=\frac{1}{f}\left[\begin{array}{cc}
0 & -1 \\
1 & 0
\end{array}\right]\left[\begin{array}{l}
x_{u} \\
y_{u}
\end{array}\right] .
$$

Factor $f$ is easily identified. Both the above systems give

$$
\operatorname{sgn} f=\operatorname{sgn}\left(x_{u} y_{v}-x_{v} y_{u}\right) ;
$$

the former and system (5.2) imply

$$
\frac{1}{f^{2}}=1+n^{2}(x, y)\left(x_{v}^{2}+y_{v}^{2}\right)
$$

the latter and system (5.2) imply

$$
f^{2}=1-n^{2}(x, y)\left(x_{u}^{2}+y_{u}^{2}\right) .
$$

Another arrangement reads

$$
\left[\begin{array}{l}
x_{u} \\
x_{v}
\end{array}\right]=\left[\begin{array}{cc}
0 & f \\
-1 / f & 0
\end{array}\right]\left[\begin{array}{l}
y_{u} \\
y_{v}
\end{array}\right], \quad\left[\begin{array}{l}
y_{u} \\
y_{v}
\end{array}\right]=\left[\begin{array}{cc}
0 & -f \\
1 / f & 0
\end{array}\right]\left[\begin{array}{l}
x_{u} \\
x_{v}
\end{array}\right]
$$


- two Bäcklund transformations, inverse of one another. The former and system (5.2) imply

$$
f^{2}=\frac{1-n^{2}(x, y) y_{u}^{2}}{1+n^{2}(x, y) y_{v}^{2}}
$$

the latter and system (5.2) imply

$$
f^{2}=\frac{1-n^{2}(x, y) x_{u}^{2}}{1+n^{2}(x, y) x_{v}^{2}}
$$

The integrability conditions, which pertain to the Bäcklund transformations in hand, read

$$
\left[\frac{\partial}{\partial u} \frac{\partial}{\partial v}\right]\left[\begin{array}{cc}
1 / f & 0 \\
0 & f
\end{array}\right]\left[\begin{array}{ll}
x_{u} & y_{u} \\
x_{v} & y_{v}
\end{array}\right]=0
$$

and result in equation (5.7) after algebraic manipulations.

Equations (5.5) and (5.6) are consistent with one another and with the early equations (1.3) and (1.5), as Proposition (ii) and its proof show.

The proof is complete.

5.2. In view of the discussion above, problem (i)-(iv) stated in Section 4 amounts to looking for solutions $[x y]$ to the partial differential system

$$
\begin{gathered}
\frac{\partial}{\partial v}\left[\begin{array}{l}
x \\
y
\end{array}\right]=\frac{1}{f}\left[\begin{array}{cc}
0 & -1 \\
1 & 0
\end{array}\right] \frac{\partial}{\partial u}\left[\begin{array}{l}
x \\
y
\end{array}\right], \\
\operatorname{sgn} f=\operatorname{sgn} \kappa, f^{2}=1-n^{2}(x, y)\left(x_{u}^{2}+y_{u}^{2}\right),
\end{gathered}
$$

that is defined either in the half-strip

$$
a<u<b, 0<v<\infty
$$

or in an appropriate bounded subset of it, and satisfies the initial conditions

$$
x(u, 0)=\alpha(u), \quad y(u, 0)=\beta(u) \text { for } a \leq u \leq b .
$$

In the remaining part of the paper we will concentrate on such a problem. A behavior of solutions $[x y]$ to (5.9) and (5.10) as $v$ is close to 0 is fixed up in the next section. An algorithm for computing the same solutions is offered in Section 8. Section 9 is devoted to an example. 


\section{R. Magnanini and G. Talenti}

\section{Behavior near the caustic}

The state of affairs causes any solution of (5.9) and (5.10) to suffer from singularities near the initial line — indeed,

$$
x_{v}^{2}(u, v)+y_{v}^{2}(u, v) \rightarrow \infty
$$

as $a \leq u \leq b$ and $v$ approaches 0 . The proposition below offers more details on the subject, as well as a proof of expansions (4.6).

Proposition 6.1. Let $x$ and $y$ obey system (5.9) and initial conditions (5.10). Assume $x(u, v)$ and $y(u, v)$ depend smoothly upon $u$ for every nonnegative, sufficiently small $v$. Then the following asymptotic expansion holds:

$\left[\begin{array}{l}x(u, v) \\ y(u, v)\end{array}\right]=\left[\begin{array}{c}\alpha(u) \\ \beta(u)\end{array}\right]+\frac{(3 v)^{2 / 3} \operatorname{sgn} \kappa(u)}{2|\kappa(u)|^{1 / 3} \sqrt{\alpha^{\prime}(u)^{2}+\beta^{\prime}(u)^{2}}}\left[\begin{array}{c}-\beta^{\prime}(u) \\ \alpha^{\prime}(u)\end{array}\right]+o\left(v^{2 / 3}\right)$ as $a \leq u \leq b$ and $v$ is positive and approaches 0 .

Proof. Equation (3.2), equations (5.9) and initial conditions (5.10) tell us that

$$
f(u, v) \rightarrow 0
$$

as $a \leq u \leq b$ and $v$ is positive and approaches 0 . Therefore,

$$
\lim _{v \downarrow 0} \frac{v^{1 / 3}}{f(u, v)}=\lim _{v \downarrow 0} \operatorname{sgn} f(u, v)\left\{\operatorname{sgn} f(u, v) / \frac{\partial}{\partial u} f^{3}(u, v)\right\}^{1 / 3},
$$

by L'Hospital's rule. Equations (5.9) give successively

$$
\begin{gathered}
\frac{\partial}{\partial v} n^{2}(x, y)=\frac{2 n^{2}(x, y)}{f(u, v)}\left\langle\nabla \log n(x, y),\left[\begin{array}{c}
-y_{u} \\
x_{u}
\end{array}\right]\right\rangle, \\
\frac{\partial}{\partial v}\left(x_{u}^{2}+y_{u}^{2}\right)=-\frac{2}{f(u, v)}\left(x_{u} y_{u u}-x_{u u} y_{u}\right)
\end{gathered}
$$

and

$$
\begin{aligned}
\frac{\partial}{\partial v} f^{3}(u, v)=3\left(x_{u}^{2}+y_{u}^{2}\right)^{3 / 2} & n^{2}(x, y)\left\{\frac{x_{u} y_{u u}-x_{u u} y_{u}}{\left(x_{u}^{2}+y_{u}^{2}\right)^{3 / 2}}\right. \\
& \left.-\left\langle\nabla \log n(x, y),\left(x_{u}^{2}+y_{u}^{2}\right)^{-1 / 2}\left[\begin{array}{c}
-y_{u} \\
x_{u}
\end{array}\right]\right\rangle\right\} .
\end{aligned}
$$

We infer

$$
\lim _{v \downarrow 0}(3 v)^{1 / 3}\left[\begin{array}{l}
x_{v} \\
y_{v}
\end{array}\right]=\frac{\operatorname{sgn} \kappa(u)}{|\kappa(u)|^{1 / 3} \sqrt{\alpha^{\prime}(u)^{2}+\beta^{\prime}(u)^{2}}}\left[\begin{array}{c}
-\beta^{\prime}(u) \\
\alpha^{\prime}(u)
\end{array}\right]
$$

because of (4.3) and (4.4). The conclusion follows. 


\section{Differentiating discrete and polluted data}

7.1. The present section is devoted to an auxiliary technique, which is a key to our main results.

Differentiating a real-valued function of one real variable is among the most elementary processes of mathematical and numerical analysis, but is also a significant prototype of those problems that are nowadays qualified ill-posed in the sense of Hadamard. On the other hand, differentiations with respect to those directions, which are tangent to the initial surface, are the main source of the ill-posed character of initial value problems for elliptic systems of partial differential equations. In the next section we tune a numerical approach to one of such problems, where a suitable approximation of tangential derivatives plays an important role.

Methods of approximating derivatives of smooth functions under nonexact data have been widely experimented over the years. Here we take the opportunity of sketching one more of such methods. We consider the case where data consist of discrete and noisy samples, nodes are equally spaced, and information is available on both the relevant noise and the underlying smoothness. Our method is inspired by ideas that the theory of statistical learning has recently revived — see e.g. [45], [65], [198]-[201], [217]-[220] - and of course mimics several of its ascendants - see e.g. [2], [3], [7]-[8], [9], [13], [19], [38], [44], [47], [48], [51], [52], [59], [73], [79], [97], [103], [110], [111], [133], [134], [146], [155]-[156], [157], [158], [162], [180]-[181], [182], [188], [193], [203], [204], [216], [225].

7.2. Items in input include:

(i) the end points of a bounded interval $-a$ and $b$;

(ii) the number of both nodes and samples - an integer $N$, larger than 2;

(iii) equally spaced nodes from $a$ to $b$ - specifically,

$$
x_{k}=a+(k-1) \frac{b-a}{N-1} \quad(k=1, \ldots, N) ;
$$

(iv) noisy samples - a sequence

$$
g_{1}, g_{2}, \ldots, g_{N}
$$

of real numbers whatever.

Goals include recovering some noiseless function $f$ and the derivative $f^{\prime}$ of $f$ based upon the following information only:

(v) $f$ is smooth;

(vi) $f\left(x_{k}\right)$ is close to $g_{k}$, for $k=1,2, \ldots, N$. 


\section{R. Magnanini and G. Talenti}

Our recipe segments into the following three steps. First, let $\lambda$ and $\mu$ be positive parameters, and solve the variational problem

$$
\sum_{k=1}^{N}\left[u\left(x_{k}\right)-g_{k}\right]^{2}+\lambda \int_{-\infty}^{\infty}\left[\mu^{7}\left(u^{\prime \prime \prime \prime}\right)^{2}+\mu^{-1} u^{2}\right] d x=\text { minimum }
$$

under the condition

$$
u \text { belongs to Sobolev space } W^{4,2}(-\infty, \infty) .
$$

Second, adjust $\lambda$ and $\mu$ properly. Third, take $u, u^{\prime}$ as approximations of $f$, $f^{\prime}$.

Observe that

$$
\sum_{k=1}^{N}\left[u\left(x_{k}\right)-g_{k}\right]^{2}
$$

the beginning of line (7.1), is a data-fidelity term;

$$
\int_{-\infty}^{\infty}\left[\mu^{7}\left(u^{\prime \prime \prime \prime}\right)^{2}+\mu^{-1} u^{2}\right] d x
$$

a squared and conveniently scaled norm of $u$ in $W^{4,2}(-\infty, \infty)$, plays the role of a penalty. Parameter $\lambda$ balances the data-fidelity term and the penalty term; parameter $\mu$ balances

$$
\int_{-\infty}^{\infty}\left(u^{\prime \prime \prime \prime}\right)^{2} d x \text { and } \int_{-\infty}^{\infty} u^{2} d x
$$

hence tunes a Rayleigh quotient of $u$.

It could be shown that the above recipe has the potential of recovering not only $f$ and $f^{\prime}$ as requested here, but $f^{\prime \prime}$ and $f^{\prime \prime \prime}$ as well. Involving

$$
\int_{-\infty}^{\infty}\left[\mu^{3}\left(u^{\prime \prime}\right)^{2}+\mu^{-1} u^{2}\right] d x
$$

instead, and letting $W^{2,2}(-\infty, \infty)$ be the space of competing functions, would be enough for our present purposes; whereas involving

$$
\int_{-\infty}^{\infty}\left[\mu\left(u^{\prime}\right)^{2}+\mu^{-1} u^{2}\right] d x
$$


and Sobolev space $W^{1,2}(-\infty, \infty)$ would not. Powers different from squares could be allowed, but would make the method impracticable.

7.3. Effective formulas read as follows. Rudiments of the calculus of variations demonstrate that Problem (7.1) \& (7.2) possesses a unique solution; moreover that such a solution — named $u$ throughout — obeys

$$
\lambda\left(\mu^{7} \frac{d^{8} u}{d x^{8}}+\mu^{-1} u\right)+\sum_{k=1}^{N}\left[u\left(x_{k}\right)-g_{k}\right] \delta\left(x-x_{k}\right)=0
$$

for $-\infty<x<\infty$. The following features are decisive: equation (7.3) is affine;

$$
\mu^{7} \frac{d^{8}}{d x^{8}}+\mu^{-1}
$$

is a positive operator in $L^{2}(-\infty, \infty)$, whose inverse mollifies;

$$
\sum_{k=1}^{N}\left[u\left(x_{k}\right)-g_{k}\right] \delta\left(\cdot-x_{k}\right)
$$

is a spike train or a shah-function. (Shah stands for a letter of Cyrillic alphabet, Ш, which is suggestive of an assemblage of vertical needles.)

Condition (7.2) and equation (7.3) give

$$
-\lambda u(x)=\sum_{k=1}^{N}\left[u\left(x_{k}\right)-g_{k}\right] K\left(\frac{x-x_{k}}{\mu}\right)
$$

for $-\infty<x<\infty$. Equation (7.4) gives

$$
A\left[\begin{array}{c}
u\left(x_{1}\right) \\
\vdots \\
u\left(x_{N}\right)
\end{array}\right]+\lambda\left[\begin{array}{c}
u\left(x_{1}\right) \\
\vdots \\
u\left(x_{N}\right)
\end{array}\right]=A\left[\begin{array}{c}
g_{1} \\
\vdots \\
g_{N}
\end{array}\right] .
$$

Here $K$ denotes an appropriate reproducing kernel for the Sobolev space $W^{4,2}(-\infty, \infty)$ - in other words a fundamental solution to $d^{8} / d x^{8}+1$. $K$ is the solution to

$$
\frac{d^{8} K}{d x^{8}}+K=\delta(x)
$$

that decays at infinity, is given by

$$
\pi K(x)=\int_{0}^{\infty} \frac{\cos (x \xi)}{1+\xi^{8}} d \xi
$$


R. Magnanini and G. Talenti

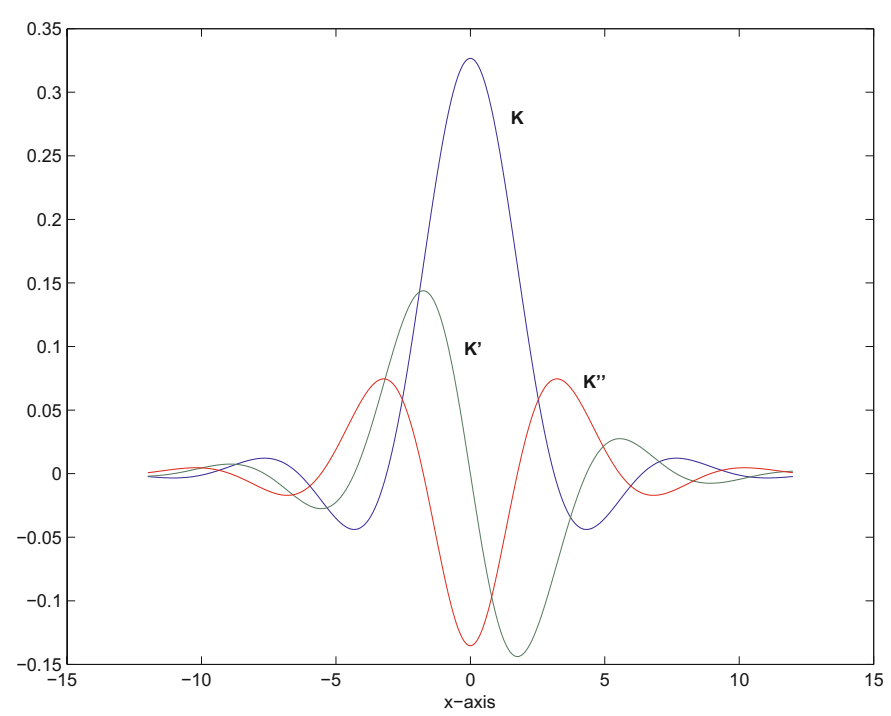

Figure 4. Plots of $K, K^{\prime}$ and $K^{\prime \prime}$.

for $-\infty<x<\infty$, is even and positive definite. Secondly,

$$
A=\left[\begin{array}{cccc}
K(0) & K\left(\frac{x_{1}-x_{2}}{\mu}\right) & \ldots & K\left(\frac{x_{1}-x_{N}}{\mu}\right) \\
K\left(\frac{x_{2}-x_{1}}{\mu}\right) & K(0) & \ldots & K\left(\frac{x_{2}-x_{N}}{\mu}\right) \\
\vdots & \vdots & \ddots & \vdots \\
K\left(\frac{x_{N}-x_{1}}{\mu}\right) & K\left(\frac{x_{N}-x_{2}}{\mu}\right) & \ldots & K(0)
\end{array}\right]
$$

- a symmetric, positive definite Toeplitz matrix.

Let $\nu=\pi / 8$. Manipulating formula (7.6) gives

$$
K(x)=\frac{1}{8 \sin \nu}-\frac{1}{8 \cos \nu} \frac{x^{2}}{2}+\frac{1}{8 \cos \nu} \frac{x^{4}}{24}-\frac{1}{8 \sin \nu} \frac{x^{6}}{720}+\frac{1}{2} \frac{|x|^{7}}{5040}+O\left(x^{8}\right)
$$

as $x$ approaches zero $-K$ behaves near zero like a spline of order seven. Calculus of residues, or convenient formulas from [PBM, Section 2.5.10], give

$$
4 K(x)=e^{-|x| \cos \nu} \cos (|x| \sin \nu-\nu)+e^{-|x| \sin \nu} \sin (|x| \cos \nu+\nu)
$$


as $-\infty<x<\infty$. Therefore,

$$
\begin{array}{r}
\frac{d^{n} K}{d x^{n}}(x)=\frac{(-\operatorname{sgn} x)^{n}}{4}\left\{e^{-|x| \cos \nu} \cos (|x| \sin \nu-(n+1) \nu)\right. \\
\left.+e^{-|x| \sin \nu} \sin (|x| \cos \nu+(n+1) \nu-n \pi / 2)\right\}
\end{array}
$$

as $x \neq 0$ and $n=1,2,3, \ldots$ Figure 4 shows plots of $K, K^{\prime}, K^{\prime \prime}$.

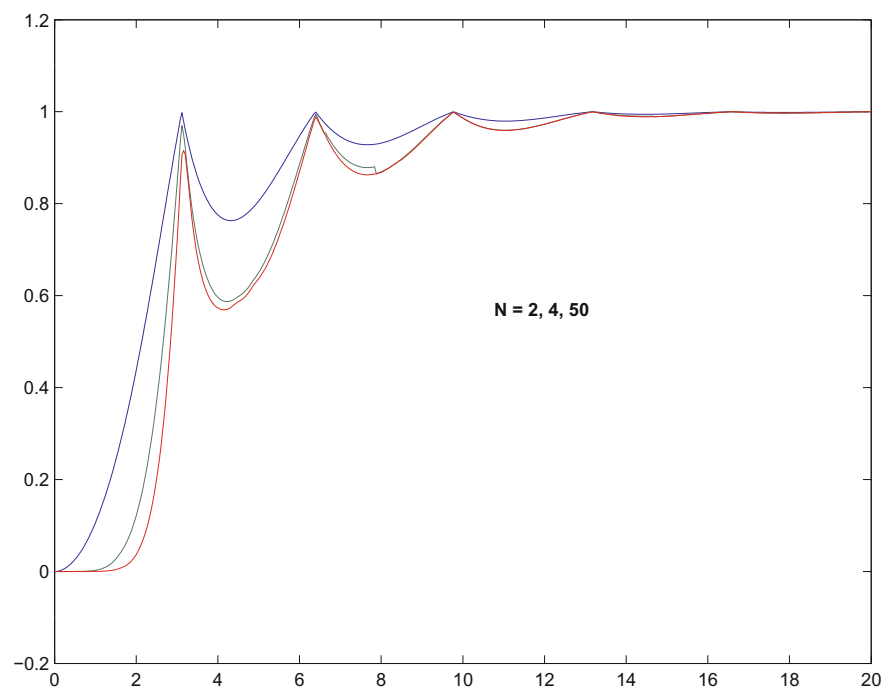

FiguRE 5. Reciprocal condition estimator of $A$, plotted versus the ratio $(b-a) /((N-1) \mu)$.

Analysis shows the following. The spectrum of $A$ lies in the open interval $] 0, N /(8 \sin \nu)[$. All eigenvalues of $A$ are close to $1 /(8 \sin \nu)$, if $\mu(N-1) /(b-a)$ is small; otherwise, the largest eigenvalue of $A$ is close to $N /(8 \sin \nu)$ and most remaining eigenvalues of $A$ are close to 0 . In particular, $A$ is invertible anyway; $A$ is either well-conditioned or ill-conditioned depending on whether $\mu(N-1) /(b-a)$ is small or large. Figure 5 shows plots of a reciprocal condition estimator of $A$ versus $(b-a) /((N-1) \mu)$.

Equation (7.4) implies that $u$ belongs to the linear span of

$$
K\left(\frac{\cdot-x_{1}}{\mu}\right), \ldots, K\left(\frac{\cdot-x_{N}}{\mu}\right)
$$

- translations and dilations of $K$. Such items own either a spike-shaped or a well-rounded profile depending on whether $\mu$ is small or large, inasmuch 


\section{R. Magnanini and G. Talenti}

as

$$
\frac{\int_{-\infty}^{\infty}|(d / d x) \operatorname{Item}|^{2} d x}{\int_{-\infty}^{\infty}|\operatorname{Item}|^{2} d x}=\frac{5}{7} \mu^{-2} \tan \nu, \frac{\int_{-\infty}^{\infty}\left|\left(d^{2} / d x^{2}\right) \operatorname{Item}\right|^{2} d x}{\int_{-\infty}^{\infty}|\operatorname{Item}|^{2} d x}=\frac{3}{7} \mu^{-4} \tan \nu .
$$

The same items are definitely linearly independent, although appropriate formulas and analysis show that their Gram matrix is well-conditioned only if $\mu(N-1) /(b-a)$ is small enough.

Equation (7.5) determines $u\left(x_{1}\right), \ldots, u\left(x_{N}\right)$ in terms of data. Note that they solve

$$
\sum_{k=1}^{N}\left[u\left(x_{k}\right)-g_{k}\right]^{2}+\lambda\left[u\left(x_{1}\right) \cdots u\left(x_{N}\right)\right] A^{-1}\left[\begin{array}{c}
u\left(x_{1}\right) \\
\vdots \\
u\left(x_{N}\right)
\end{array}\right]=\text { minimum }
$$

- a standard finite-dimensional least-square problem, where $\lambda$ and the inverse of $A$ imitate a regulating parameter à la Tikhonov and a penalty, respectively.

Now we are in a position to draw conclusions. Let Id be the $N \times N$ unit matrix, and

$$
R=(\lambda \operatorname{Id}+A)^{-1}
$$

- a resolvent. Let $B$ the vector-valued function such that

$$
B(x)=R\left[\begin{array}{c}
K\left(\frac{x-x_{1}}{\mu}\right) \\
\vdots \\
K\left(\frac{x-x_{N}}{\mu}\right)
\end{array}\right]
$$

for $-\infty<x<\infty$ - an alternative basis in the linear span mentioned above. Define $C$ and $D$ by $C=A R$ and

$$
D=\mu^{-1}\left[\begin{array}{cccc}
0 & K^{\prime}\left(\frac{x_{1}-x_{2}}{\mu}\right) & \ldots & K^{\prime}\left(\frac{x_{1}-x_{N}}{\mu}\right) \\
K^{\prime}\left(\frac{x_{2}-x_{1}}{\mu}\right) & 0 & \ldots & K^{\prime}\left(\frac{x_{2}-x_{N}}{\mu}\right) \\
\vdots & \vdots & \ddots & \vdots \\
K^{\prime}\left(\frac{x_{N}-x_{1}}{\mu}\right) & K^{\prime}\left(\frac{x_{N}-x_{2}}{\mu}\right) & \ldots & 0
\end{array}\right]
$$

$R$. 
The following equations hold.

$$
u(x)=\left[g_{1} \cdots g_{N}\right] B(x)
$$

for $-\infty<x<\infty$,

$$
\begin{aligned}
& {\left[\begin{array}{c}
u\left(x_{1}\right) \\
\vdots \\
u\left(x_{N}\right)
\end{array}\right]=C\left[\begin{array}{c}
g_{1} \\
\vdots \\
g_{N}
\end{array}\right],} \\
& {\left[\begin{array}{c}
u^{\prime}\left(x_{1}\right) \\
\vdots \\
u^{\prime}\left(x_{N}\right)
\end{array}\right]=D\left[\begin{array}{c}
g_{1} \\
\vdots \\
g_{N}
\end{array}\right] .}
\end{aligned}
$$

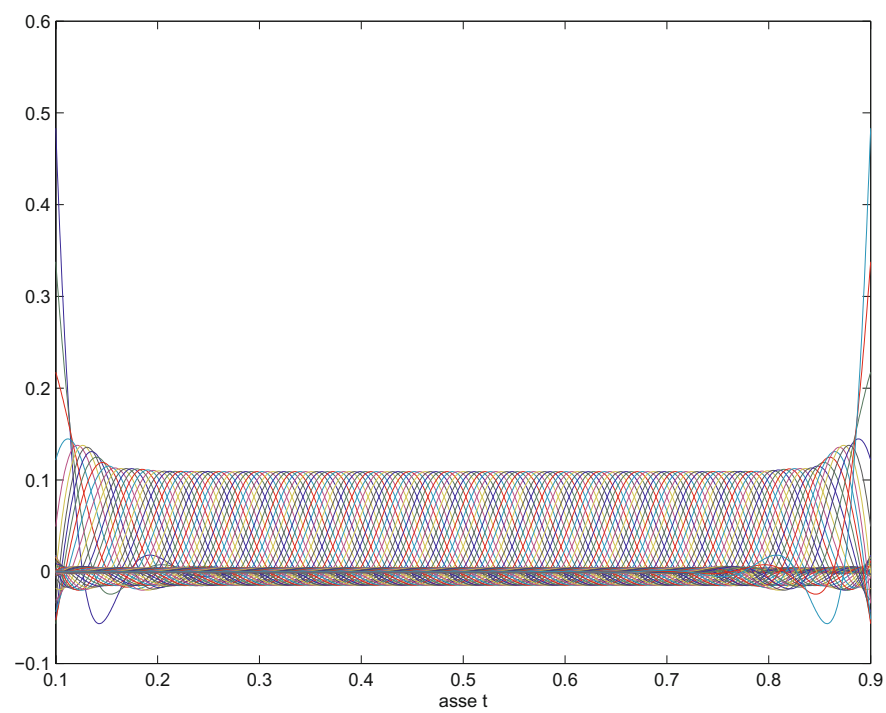

Figure 6 . The alternative basis $B$ : plots of $B_{1}(x), \ldots$, $B_{N}(x)$ versus $x$.

As figures 6, 7 and 8 show, $B$ and $C$ mimic a typical Green's function from two different perspectives, $D$ mimics a derivative of a Green's function. Observe incidentally that

$$
\begin{gathered}
\int_{-\infty}^{\infty}\left[\mu^{7}\left(B^{\prime \prime \prime \prime}\right)^{T} B^{\prime \prime \prime \prime}+\mu^{-1} B^{T} B\right] d x=\operatorname{tr}\left[A(\lambda \operatorname{Id}+A)^{-2}\right], \\
C=\operatorname{Id}-\lambda(\lambda \operatorname{Id}+A)^{-1}
\end{gathered}
$$


R. Magnanini and G. Talenti

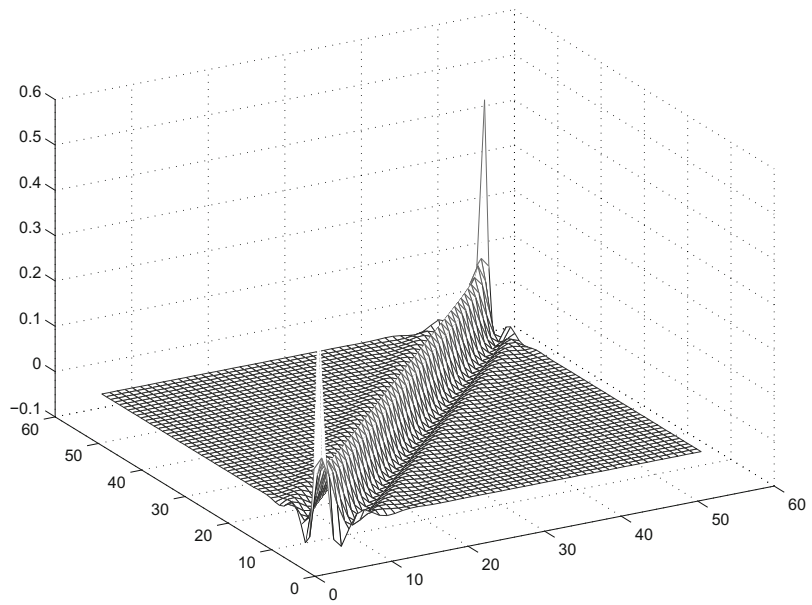

Figure 7. Matrix $C$ playing the role of a regularizing filter.

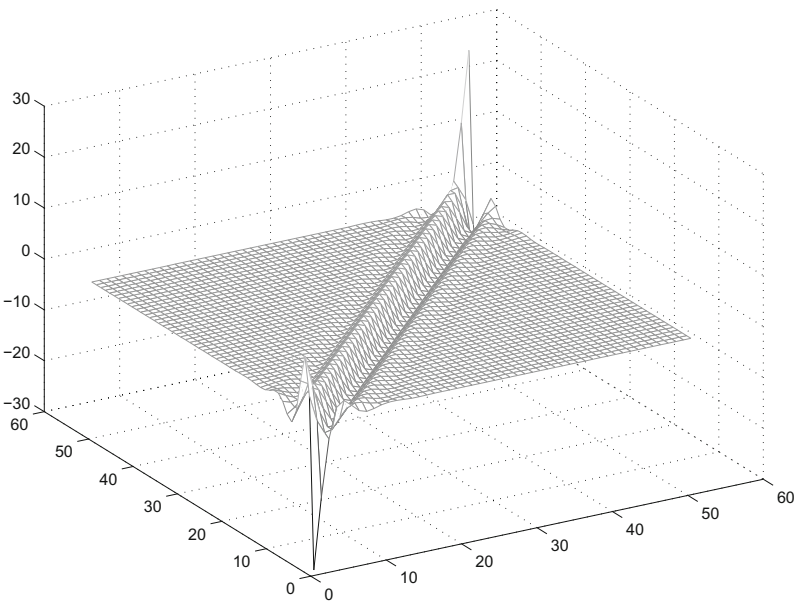

FiguRE 8. Matrix $D$, simulating differentiation.

and that $B$ and $C$ solve the variational problem

$$
\lambda \int_{-\infty}^{\infty}\left[\mu^{7}\left(B^{\prime \prime \prime \prime}\right)^{T} B^{\prime \prime \prime \prime}+\mu^{-1} B^{T} B\right] d x+\operatorname{tr}\left[\left(C^{T}-\mathrm{Id}\right)(C-\mathrm{Id})\right]=\text { minimum }
$$


subject to the conditions

$$
B \in\left[W^{4,2}(-\infty, \infty)\right]^{N}, \quad C=\left[\begin{array}{cccc}
B_{1}\left(x_{1}\right) & B_{2}\left(x_{1}\right) & \ldots & B_{N}\left(x_{1}\right) \\
B_{1}\left(x_{2}\right) & B_{2}\left(x_{2}\right) & \ldots & B_{N}\left(x_{2}\right) \\
\vdots & \vdots & \ddots & \vdots \\
B_{1}\left(x_{N}\right) & B_{2}\left(x_{N}\right) & \ldots & B_{N}\left(x_{N}\right)
\end{array}\right] .
$$

7.4. Here we offer directions for adjusting $\lambda$ and $\mu$ properly. Parameter $\lambda$ - dimensionless - discriminates whether solution $u$ to Problem (7.1) \& (7.2) either fits data well (but is simultaneously sensible of noise), or else is little affected by noise (but departs somewhat from data). In loose terms, the following statements hold. First, $u$ virtually interpolates $g_{1}, \ldots, g_{N}$ if $\lambda$ is close to zero; however, $u$ is liable to own an irregular profile at the same time. Second, $u$ quenches smoothly if $\lambda$ grows larger and larger. Indeed,

$$
\begin{gathered}
\int_{-\infty}^{\infty}\left[\mu^{7}\left(B^{\prime \prime \prime \prime}\right)^{T} B^{\prime \prime \prime \prime}+\mu^{-1} B^{T} B\right] d x=\operatorname{tr} A^{-1}+O(\lambda), \\
C=\operatorname{Id}+O(\lambda)
\end{gathered}
$$

as $\lambda$ approaches zero;

$$
\left\|d^{n} B(x) / d x^{n}\right\| \leq \lambda^{-1} \frac{\sqrt{N}}{8 \sin [(n+1) \nu]}
$$

as $-\infty<x<\infty$ and $n=0,1, \ldots, 6$.

Parameter $\mu$ - making $\mu /(b-a)$ dimensionless - determines how much $u$ is close to, or departs from a spike train. Indeed,

$$
B(x)=\mu \frac{8 \sin \nu}{1+\lambda \sin \nu}\left[\begin{array}{c}
\delta\left(x-x_{1}\right) \\
\vdots \\
\delta\left(x-x_{N}\right)
\end{array}\right]+O\left(\mu^{3}\right)
$$

as $-\infty<x<\infty$ and $\mu$ approaches zero;

$$
B(x)=\frac{1}{N+8 \lambda \sin \nu}\left[\begin{array}{c}
1 \\
\vdots \\
1
\end{array}\right]+O\left(\mu^{-2}\right)
$$

as $-\infty<x<\infty$ and $\mu$ grows larger and larger.

In the case where suitable information is available a priori, a theorem from Section 7 below suggests which values of $\lambda$ and $\mu$ work properly. Otherwise, parameter $\lambda$ may be determined based upon the discrepancy 
R. Magnanini and G. Talenti

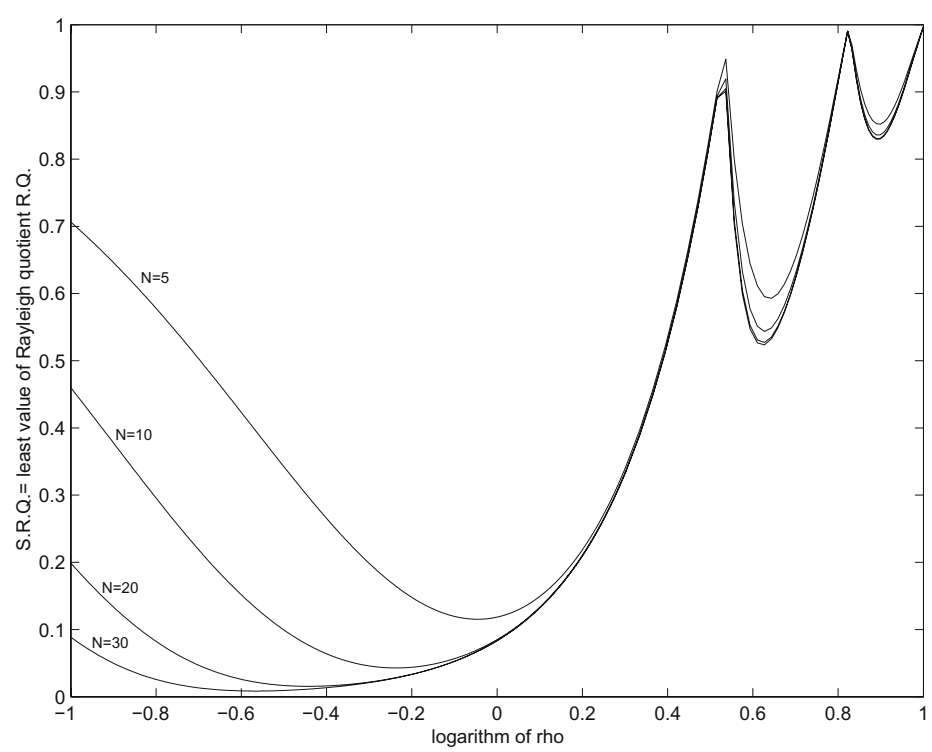

Figure 9. Plots of S.R.Q. versus $\log \rho$. Here $\rho=(b-$ a) $/((N-1) \mu)$.

principle, the cross-validation, the L-curve criterion, or other customary devices — see e.g. [86, Chapter 7] for details.

Parameter $\mu$ is expediently identified by the following recipe. Let

$$
\text { R.Q. of } v=\mu^{14} \frac{\int_{-\infty}^{\infty}\left[(d / d x)^{7} v(x)\right]^{2} d x}{\int_{-\infty}^{\infty}[v(x)]^{2} d x}
$$

be a dimensionless Rayleigh quotient; define a relevant minimum by

$$
\text { S.R.Q. }=\min \left\{\text { R.Q. of } v: 0 \neq v \in \operatorname{span} \text { of } K\left(\frac{\cdot-x_{1}}{\mu}\right), \ldots, K\left(\frac{\cdot-x_{N}}{\mu}\right)\right\} \text {; }
$$

then determine $\mu$ so that

$$
\text { S.R.Q. = minimum. }
$$

Equation (7.8) causes solution $u$ of Problem (7.1) \& (7.2) to retain its most favorable Rayleigh quotient. Equation (7.8) can be approached via equation (7.9) below and tools from linear algebra, although care must be taken of the ill-condition of the involved matrices. 
Let

$$
\begin{gathered}
G=\mu^{-1} \times \text { Gram matrix of } K\left(\frac{\cdot-x_{1}}{\mu}\right), \ldots, K\left(\frac{\cdot-x_{N}}{\mu}\right), \\
H=\mu^{-1} \times \text { Gram matrix of } K^{(7)}\left(\frac{\cdot-x_{1}}{\mu}\right), \ldots, K^{(7)}\left(\frac{\cdot-x_{N}}{\mu}\right) ;
\end{gathered}
$$

let $L$ denote the autocorrelation function of $K$, videlicet

$$
\pi L(x)=\int_{0}^{\infty}\left(1+\xi^{8}\right)^{-2} \cos (x \xi) d \xi
$$

for $-\infty<x<\infty$. We have

$$
\begin{gathered}
G=\left[L\left(\frac{x_{j}-x_{k}}{\mu}\right)\right]_{j, k=1, \ldots, N}, H=\left[-L^{(14)}\left(\frac{x_{j}-x_{k}}{\mu}\right)\right]_{j, k=1, \ldots, N} ; \\
L(x)=\frac{7}{8} K(x)-\frac{x}{8} K^{\prime}(x), \quad L^{(14)}(x)=\frac{7}{8} K^{(6)}(x)+\frac{x}{8} K^{(7)}(x)
\end{gathered}
$$

for $-\infty<x<\infty$, and

$$
\text { S.R.Q. = least eigenvalue of } H \text { with respect to } G
$$

— in other words,

$$
\text { S.R.Q. = least eigenvalue of } G^{-1 / 2} H G^{-1 / 2} \text {. }
$$

Figure 9 shows plots of S.R.Q. versus $(b-a) /((N-1) \mu)$. The following table lists sample solutions to equation (7.8).

$\begin{array}{lccc}N & \text { minimum value } & (b-a) /((N-1) \mu) & \mu /(b-a) \\ 5 & 0.115320757000 & 0.900127730000 & 0.277738360532 \\ 10 & 0.042825969700 & 0.578865888000 & 0.191946206219 \\ 20 & 0.015529762000 & 0.361101444000 & 0.145752889726 \\ 30 & 0.008487160550 & 0.271108504000 & 0.127191726235 \\ 40 & 0.005502126710 & 0.220171280000 & 0.116459447577 \\ 50 & 0.003920874640 & 0.187117585000 & 0.109065982576 \\ 60 & 0.002967795760 & 0.163520070000 & 0.103651818045 \\ 70 & 0.002342495540 & 0.145755736000 & 0.099431789245 \\ 80 & 0.001906845850 & 0.131825897000 & 0.096022315313 \\ 100 & 0.001349762400 & 0.111466361000 & 0.090619358257 \\ 120 & 0.001016274330 & 0.097183852500 & 0.086468699567 \\ 140 & 0.000798662701 & 0.086282601400 & 0.083380015062 \\ 150 & 0.000716797334 & 0.081805225800 & 0.082041328416\end{array}$




\section{R. Magnanini and G. Talenti}

The formula

$$
\frac{\mu}{b-a} \approx 0.0415+0.5416 \times N^{-1 / 2}-0.6426 \times N^{-1}+1.3706 \times N^{-3 / 2}
$$

gives an effectual estimate of such solutions.

7.5. The following theorem holds.

Theorem 7.1. Suppose u solves problem (7.1) \& (7.2); suppose $f, \varepsilon$ and $E$ obey

$$
\begin{gathered}
\left|f\left(x_{k}\right)-g_{k}\right| \leq \varepsilon \quad(k=1, \ldots, N) \\
\mu^{-4} \int_{-\infty}^{\infty} f(x)^{2} d x+\mu^{4} \int_{-\infty}^{\infty}\left[f^{\prime \prime \prime \prime}(x)\right]^{2} d x \leq 2(b-a)^{-3} E^{2}
\end{gathered}
$$

let

$$
\delta=\max \left\{(1-1 / N)^{-1 / 2} \frac{\varepsilon}{E},(N-1)^{-2}\right\} .
$$

If $\delta$ approaches zero, and

$$
\frac{\lambda}{N}\left(\frac{\mu}{b-a}\right)^{3}\left(\frac{\varepsilon}{E}\right)^{-2}
$$

is bounded and bounded away from zero, then

$$
\begin{gathered}
E^{-1} \max \{|f(x)-u(x)|: a \leq x \leq b\}=O\left(\delta^{3 / 4}\right), \\
E^{-1}(b-a) \max \left\{\left|f^{\prime}(x)-u^{\prime}(x)\right|: a \leq x \leq b\right\}=O\left(\delta^{1 / 4}\right) .
\end{gathered}
$$

Proof. Let

$$
v=f-u \text {. }
$$

The functional $J$, whose domain is $W^{4,2}(-\infty, \infty)$ and whose value at any trial function $\varphi$ obeys

$$
J(\varphi)=\sum_{k=1}^{N}\left[\varphi\left(x_{k}\right)-g_{k}\right]^{2}+\lambda \int_{-\infty}^{\infty}\left[\mu^{7}\left(\varphi^{\prime \prime \prime \prime}\right)^{2}+\mu^{-1} \varphi^{2}\right] d x,
$$

attains its minimum value at $u$. Consequently,

$$
\begin{gathered}
J(f)=J(u)+(\text { a remainder }) \\
\text { remainder }=\sum_{k=1}^{N}\left[v\left(x_{k}\right)\right]^{2}+\lambda \int_{-\infty}^{\infty}\left[\mu^{7}\left(v^{\prime \prime \prime \prime}\right)^{2}+\mu^{-1} v^{2}\right] d x .
\end{gathered}
$$


Since

$$
\begin{gathered}
J(f) \leq N \varepsilon^{2}+2 \lambda\left(\frac{\mu}{b-a}\right)^{3} E^{2}, \\
J(u) \geq 0 \\
\int_{-\infty}^{\infty}\left[\mu^{7}\left(v^{\prime \prime \prime \prime}\right)^{2}+\mu^{-1} v^{2}\right] d x \geq 2 \mu^{3} \int_{-\infty}^{\infty}\left(v^{\prime \prime}\right)^{2} d x,
\end{gathered}
$$

we infer

$$
\sum_{k=1}^{N}\left[v\left(x_{k}\right)\right]^{2}+2 \lambda \mu^{3} \int_{-\infty}^{\infty}\left(v^{\prime \prime}\right)^{2} d x \leq E^{2}\left\{N\left(\frac{\varepsilon}{E}\right)^{2}+2 \lambda\left(\frac{\mu}{b-a}\right)^{3}\right\} .
$$

Combining inequality (7.12) and Lemma 7.2 below results in

$$
\begin{aligned}
& (b-a)^{-1} \int_{a}^{b} v^{2} d x \\
& \leq E^{2}\left\{\frac{N}{N-1}+\frac{1}{\pi^{4}(N-1)^{4}} \frac{N}{2 \lambda}\left(\frac{\mu}{b-a}\right)^{-3}\right\}\left\{\left(\frac{\varepsilon}{E}\right)^{2}+\frac{2 \lambda}{N}\left(\frac{\mu}{b-a}\right)^{3}\right\} ;
\end{aligned}
$$

inequality (7.12) also yields

$$
(b-a)^{3} \int_{a}^{b}\left(v^{\prime \prime}\right)^{2} d x \leq E^{2}\left\{1+\frac{N}{2 \lambda}\left(\frac{\mu}{b-a}\right)^{-3}\left(\frac{\varepsilon}{E}\right)^{2}\right\} .
$$

The last two inequalities and a hypothesis imply

$$
\begin{gathered}
(b-a)^{-1} \int_{a}^{b} v^{2} d x \leq \text { (Const.) } E^{2} \delta^{2}, \\
(b-a)^{3} \int_{a}^{b}\left(v^{\prime \prime}\right)^{2} d x \leq \text { (Const.) } E^{2} .
\end{gathered}
$$

The conclusions follow from (7.11), (7.13) and (7.14), by virtue of Lemma 7.3 below.

Lemma 7.2. Let $-\infty<a<b<\infty$, and $N=2,3, \ldots$; let

$$
\Delta x=\frac{b-a}{N-1}, \quad x_{k}=a+(k-1) \Delta x \quad(k=1, \ldots, N) .
$$


R. Magnanini and G. Talenti

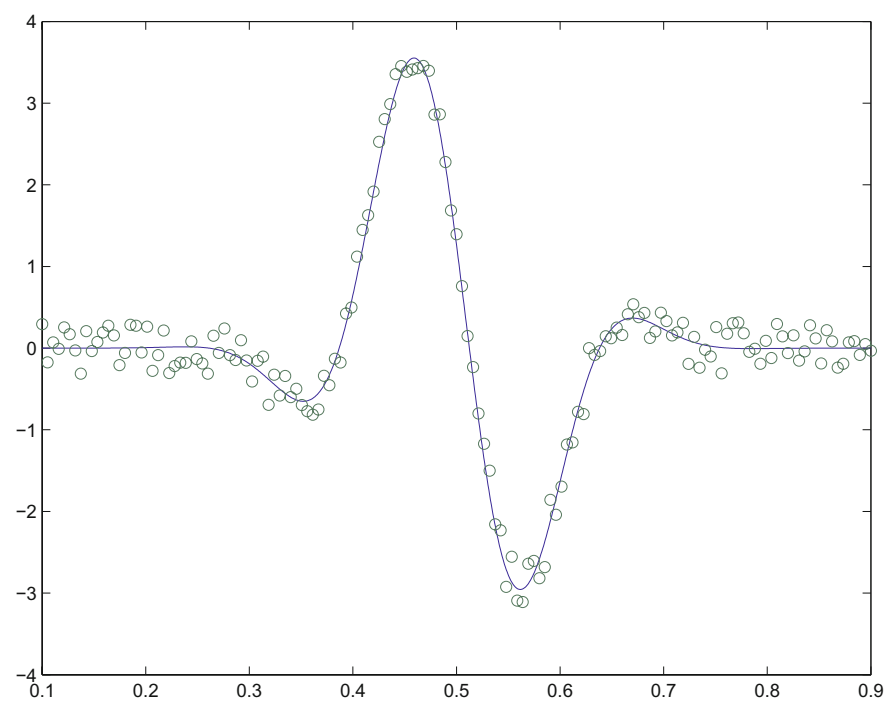

FIgURE 10. Original function (dotted), and samples polluted by noise (circled).

The inequality

$$
\left\{\int_{a}^{b} v^{2} d x\right\}^{1 / 2} \leq(\Delta x)^{-1 / 2}\left\{\sum_{k=1}^{N} v\left(x_{k}\right)^{2} d x\right\}^{1 / 2}+\left(\frac{\Delta x}{\pi}\right)^{2}\left\{\int_{a}^{b}\left(v^{\prime \prime}\right)^{2} d x\right\}^{1 / 2}
$$

holds for every $v$ from $W^{2,2}(a, b)$.

Lemma 7.3. Suppose $v$ is in $W^{2,2}(a, b)$, and

$$
(b-a)^{-1 / 2}\left\{\int_{a}^{b} v^{2} d x\right\}^{1 / 2}=\|v\|, \quad(b-a)^{3} \int_{a}^{b}\left(v^{\prime \prime}\right)^{2} d x=1 .
$$

The following inequalities hold:

$$
\begin{gathered}
\max |v| \leq 2^{1 / 4} 3^{-3 / 8}\|v\|^{3 / 4}+O(\|v\|), \\
(b-a) \max \left|v^{\prime}\right| \leq 2^{1 / 4} 3^{-3 / 8}\|v\|^{1 / 4}+O(\|v\|) .
\end{gathered}
$$

The proofs of Lemma 7.2 and 7.3 are beyond the scope of the presen paper, and are omitted. 
On Complex-valued 2D Eikonals IV

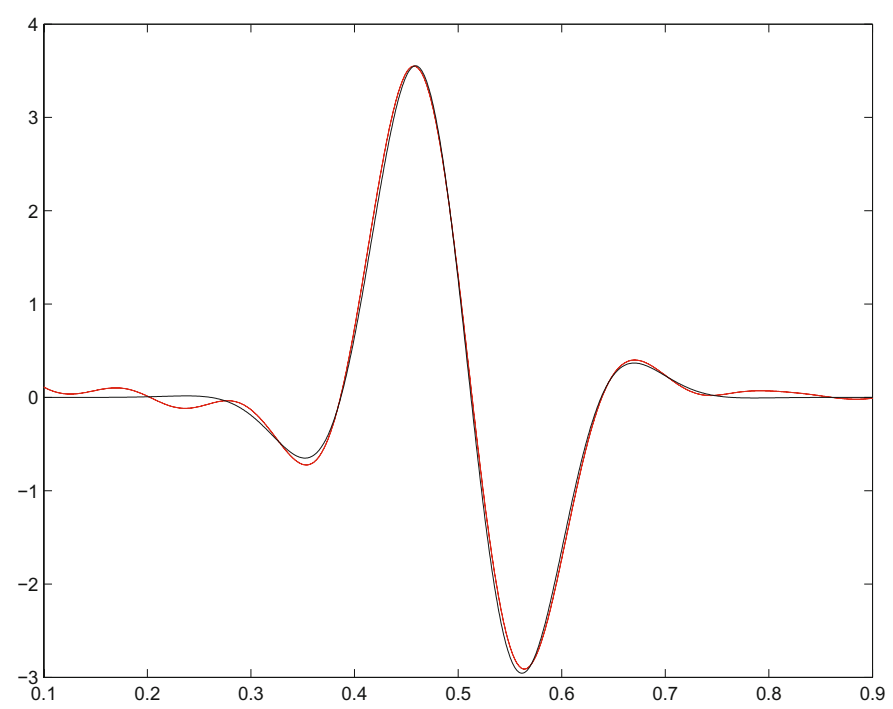

FiguRE 11. Original and recovered functions.

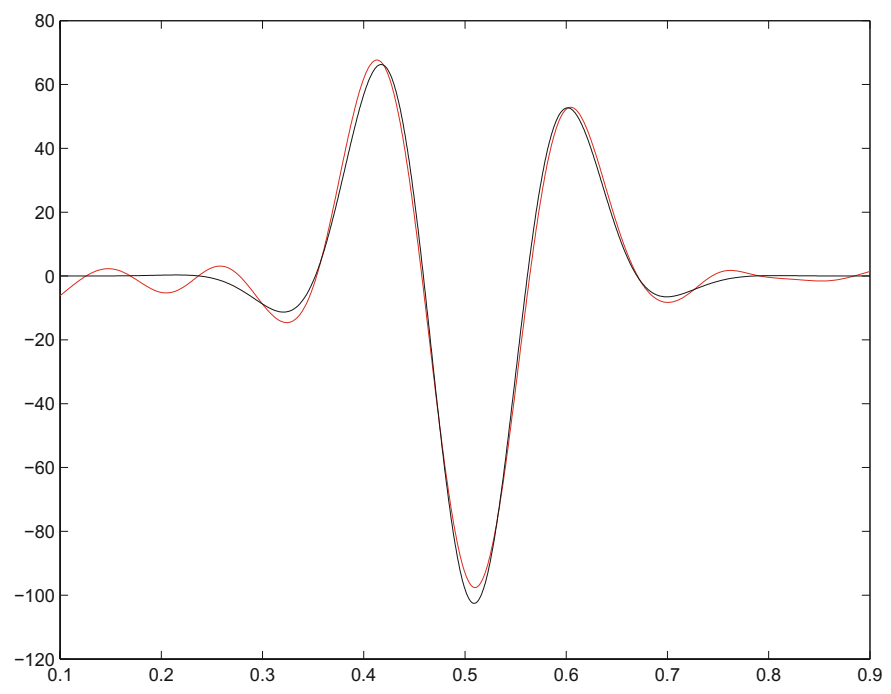

FiguRE 12. Original and recovered derivatives.

7.6. Here is an example, demonstrating how the present method works. Let

$$
\begin{gathered}
a=0, b=1, \\
\left.f(x)=\exp \left[-72\left(x-\frac{1}{2}\right)^{2}\right)\right][\cos (25 x)-4 \sin (25 x)],
\end{gathered}
$$




$$
\begin{gathered}
N=150, \\
g_{k}=f\left(x_{k}\right) \pm(5 \% \text { random noise }) \quad(k=1, \ldots, N) .
\end{gathered}
$$

Let parameter $\lambda$ obey

$$
\lambda=10^{-6},
$$

let parameter $\mu$ be given by formula (7.10), and let $u$ solve problem (7.1) $\&$ (7.2). Figure 10 plots $g_{1}, g_{2}, \ldots, g_{N}$ versus $x_{1}, x_{2}, \ldots, x_{N}$; figure 11 plots $f$ and $u$, and figure 12 plots $f^{\prime}$ and $u^{\prime}$.

\section{Discrete setting}

8.1. Rendering problem (5.9) and (5.10) into an effective discrete form entails coping with singularities of solutions, overflows, and ill-posedness in the sense of Hadamard.

Singularities result from features of both the system and the initial conditions in hand, as already remarked in Section 6. Overflows take place whenever solutions stop to obey the constraint

$$
n^{2}(x, y)\left(x_{u}^{2}+y_{u}^{2}\right)<1 .
$$

Note that the system

$$
\frac{\partial}{\partial v}\left[\begin{array}{l}
x \\
y
\end{array}\right]=\left[\begin{array}{cc}
0 & 1 \\
-1 & 0
\end{array}\right] \frac{\partial}{\partial u}\left[\begin{array}{l}
x \\
y
\end{array}\right]
$$

— Cauchy-Riemann, a possible linearized version of (5.9) — possesses obvious solutions

$$
\left[\begin{array}{l}
x \\
y
\end{array}\right]=\exp (-\sqrt{t}+t v)\left[\begin{array}{c}
\cos (t u) \\
\sin (t u)
\end{array}\right]
$$

which are highly instable, i.e.

$$
\sup \left\{\left[\frac{\partial^{j} x}{\partial u^{j}}(u, 0)\right]^{2}+\left[\frac{\partial^{j} y}{\partial u^{j}}(u, 0)\right]^{2}:-\infty<u<\infty\right\} \rightarrow 0
$$

as $t \uparrow \infty$ and $j=1,2, \ldots$, and

$$
\inf \left\{x^{2}(u, v)+y^{2}(u, v):-\infty<u<\infty\right\} \rightarrow \infty
$$

as $t \uparrow \infty$ and $v$ is positive.

Ill-posedness is a typical drawback of initial value problems for partial differential equations and systems of elliptic type. It was observed by Hadamard [82]-[83], and deeply investigated by John [94]-[96], Lavrentiev jr. [120]-[122], [127], Miller [147]-[148], Payne [163]-[166] and [171]-[172], 
Pucci [176]-[179], and Tikhonov [206]-[210]. Classical surveys on the subject have been authored by Lavrentiev jr., [123], [126], Payne [167], and Tikhonov [211]. Related information is in [19]-[20], [22], [15], [92], [93], [154], [159], [189] and [205]. A sample of more recent contributions includes [5], [6], [12], [17], [21], [16], [18], [29], [24], [30], [32], [36], [33], [34], [43], [46], [49], [62]-[63], [66]-[67], [84], [85], [89], [108], [109], [127], [132], [142]-[143], [153], [160]-[161], [168]-[170], [173], [183], [185], [186], [191], [202], [212], [213], [222], [224].

One might attempt to contend with ill-posedness via theorems involving a priori bounds on solutions and similar devices. We opt not to touch on this issue in the present paper, and focus our attention on constructive aspects instead.

We rely upon: (i) asymptotic expansions, describing how relevant solutions behave near the initial line (see Section 6); (ii) a technique of approximate differentiation, especially designed for working in presence of errors (see Section 7); (iii) an appropriate injection of artificial viscosity, which softens a coefficient and protects against overflows; (iv) an imitation of the quasi-reversibility method.

8.2. Besides data exposed to view, our method takes seven parameters in input: $L, M, N, \lambda, \mu, \nu, \xi$. The first one stands for the expected span of the solution domain. Parameters number two and three are large integers that specify the number of samples in hand - e.g. $M=N=100$. The remaining four parameters set the tone of smoothing processes: $\lambda$ and $\mu$ relate to approximate differentiation; $\nu$ stands for viscosity; $\xi$ relates to quasireversibility. Of course, a priori information (such as the smoothness of solutions, and the expected location of their singularities) are momentous for guessing effectual values of these parameters.

8.3. The equations

$$
\begin{array}{ll}
\text { ustep }=(b-a) /(M-1), & \text { vstep }=L /(N-1), \\
u_{j}=a+(j-1) \text { ustep }(j=1, \ldots, M), & v_{k}=(k-1) \text { vstep }(k=1, \ldots, N),
\end{array}
$$

will be in force throughout this section. We choose a mesh to consist of the points

$$
\left(u_{j}, v_{k}\right) \quad(j=1, \ldots, M ; k=1, \ldots, N),
$$

and store sample values at mesh points in the matrices

$$
[x(j, k)]_{j=1, \ldots, M ; k=1, \ldots, N}, \quad[y(j, k)]_{j=1, \ldots, M ; k=1, \ldots, N} .
$$




\section{R. Magnanini and G. Talenti}

The columns of these matrices, i.e.

$$
x(\cdot, 1), x(\cdot, 2), \ldots, x(\cdot, N), \quad y(\cdot, 1), y(\cdot, 2), \ldots, y(\cdot, N),
$$

are recursively generated in the following way.

\section{First step.}

$$
x(j, 1)=\alpha\left(u_{j}\right), y(j, 1)=\beta\left(u_{j}\right) \quad(j=1, \ldots, M),
$$

according to initial conditions (5.10).

\section{Second step.}

$$
\begin{aligned}
& x(j, 2)=x(j, 1)+\frac{\operatorname{sgn} \kappa\left(u_{j}\right)}{2\left|\kappa\left(u_{j}\right)\right|^{1 / 3}} \frac{-\beta^{\prime}\left(u_{j}\right)}{\sqrt{\alpha^{\prime}\left(u_{j}\right)^{2}+\beta^{\prime}\left(u_{j}\right)^{2}}}\left(3 v_{2}\right)^{2 / 3}, \\
& y(j, 2)=y(j, 1)+\frac{\operatorname{sgn} \kappa\left(u_{j}\right)}{2\left|\kappa\left(u_{j}\right)\right|^{1 / 3}} \frac{\alpha^{\prime}\left(u_{j}\right)}{\sqrt{\alpha^{\prime}\left(u_{j}\right)^{2}+\beta^{\prime}\left(u_{j}\right)^{2}}}\left(3 v_{2}\right)^{2 / 3}
\end{aligned}
$$

$(j=1, \ldots, M)$, according to Proposition 6.1.

Further steps. For $k=3, \ldots, N$ do actions (i)-(iii) below.

(i) Mimic tangent differentiation $\partial / \partial u$. Avoid finite differences, use the following formulas instead:

$$
\begin{gathered}
X=x(\cdot, k-1), Y=y(\cdot, k-1), \\
A=D X, B=D Y .
\end{gathered}
$$

Here

$$
\begin{gathered}
\lambda=\text { a dimensionless positive parameter, } \\
\frac{\mu}{b-a} \simeq 0.0415+0.5416 \times M^{-1 / 2}-0.6426 \times M^{-1}+1.3706 \times M^{-3 / 2},
\end{gathered}
$$

according to Section 7;

$$
\begin{gathered}
\nu=\pi / 8, \\
4 K(u)=e^{-|u| \cos \nu} \cos (|u| \sin \nu-\nu)+e^{-|u| \sin \nu} \sin (|u| \cos \nu+\nu),
\end{gathered}
$$

and

$$
\begin{aligned}
4 K^{\prime}(u)=\operatorname{sgn}(u)\left\{-e^{-|u| \cos \nu} \cos (|u| \sin \nu\right. & -2 \nu) \\
& \left.+e^{-|u| \sin \nu} \cos (|u| \cos \nu+2 \nu)\right\}
\end{aligned}
$$

for every $u$; moreover

$$
D=\frac{1}{\mu}\left[K^{\prime}\left(\frac{u_{i}-u_{j}}{\mu}\right)\right]_{i, j=1, \ldots, M}\left\{\lambda \mathrm{Id}+\left[K\left(\frac{u_{i}-u_{j}}{\mu}\right)\right]_{i, j=1, \ldots, M}\right\}^{-1} .
$$


(ii) Enter viscosity. Modify an uncomfortable coefficient as follows:

$$
f(j)=P\left(\nu, n(X(j), Y(j)) \sqrt{A(j)^{2}+B(j)^{2}} \operatorname{sgn} \kappa\left(u_{j}\right) \quad(j=1, \ldots, M),\right.
$$

and let

$$
\begin{aligned}
U & =-\left[\begin{array}{cccc}
f(1) & 0 & \ldots & 0 \\
0 & f(2) & \ldots & 0 \\
\vdots & \vdots & \ddots & \vdots \\
0 & 0 & \ldots & f(M)
\end{array}\right]^{-1} B \\
V & =\left[\begin{array}{cccc}
f(1) & 0 & \ldots & 0 \\
0 & f(2) & \ldots & 0 \\
\vdots & \vdots & \ddots & \vdots \\
0 & 0 & \ldots & f(M)
\end{array}\right]^{-1} A
\end{aligned}
$$

Here

$$
0<\nu=\text { artificial viscosity } \leq \pi / 2,
$$

and $P$ is given by

$$
P(\nu, \rho)= \begin{cases}\sqrt{1-\rho^{2}} & \text { if } 0 \leq \rho \leq\left(1+\sin ^{2} \nu\right)^{-1 / 2}, \\ \frac{\sin \nu}{\rho+\sqrt{\rho^{2}-\cos ^{2} \nu}} & \text { if } \rho>\left(1+\sin ^{2} \nu\right)^{-1 / 2}\end{cases}
$$

- observe that

$0 \leq \rho \mapsto P(\nu, \rho)$ is strictly positive and continuously differentiable,

$P(\nu, \rho)$ approaches $\sqrt{1-\rho^{2}}$ uniformly as $0 \leq \rho \leq 1$ and $\nu$ approaches 0 .

(iii) Enter quasi-reversibility. Improve the conventional formulas

$$
x(\cdot, k)=X+\text { vstep } U, \quad y(\cdot, k)=Y+\text { vstep } V,
$$

as follows:

$$
x(\cdot, k)=\varphi\left(v_{k}\right), \quad y(\cdot, k)=\psi\left(v_{k}\right) .
$$

Here

$$
\begin{gathered}
\xi=\text { a dimensionless positive parameter; } \\
R=(\text { ustep })^{-2}\left[\begin{array}{cccccc}
2 & -5 & 4 & -1 & \cdots & 0 \\
1 & -2 & 1 & 0 & \cdots & 0 \\
\ddots & \ddots & \ddots & \ddots & \ddots & \ddots \\
0 & \cdots & 0 & 1 & -2 & 1 \\
0 & \cdots & -1 & 4 & -5 & 2
\end{array}\right]
\end{gathered}
$$




\section{R. Magnanini and G. Talenti}

a caricature of a second-order derivative; $\varphi$ is the vector-valued mappin. that obeys both the boundary conditions

$$
\varphi\left(v_{k-1}\right)=X, \quad \varphi^{\prime}\left(v_{k-1}\right)=U
$$

and a caricature of the variational constraint

$$
\int_{v_{k-1}}^{v_{k}}\left\{(\text { vstep })^{3}\left\|\varphi^{\prime \prime}(v)\right\|^{2}+3 \xi\|R \varphi(v)\|^{2}\right\} d v=\text { minimum }
$$

namely

$$
3 \xi\left\|R \varphi\left(v_{k}\right)\right\|^{2}+(v s t e p)^{2} \int_{v_{k-1}}^{v_{k}}\left\|\varphi^{\prime \prime}(v)\right\|^{2} d v=\text { minimum }
$$

$\psi$ obeys

$$
\begin{gathered}
\psi\left(v_{k-1}\right)=Y, \quad \psi^{\prime}\left(v_{k-1}\right)=V \\
3 \xi\left\|R \psi\left(v_{k}\right)\right\|^{2}+(v s t e p)^{2} \int_{v_{k-1}}^{v_{k}}\left\|\psi^{\prime \prime}(v)\right\|^{2} d v=\text { minimum. }
\end{gathered}
$$

As is easy to see, the differential equations

$$
\varphi^{\prime \prime \prime \prime}(v)=0 \text { and } \psi^{\prime \prime \prime \prime}(v)=0 \text { if } v_{k-1}<v<v_{k}
$$

and the extra boundary conditions

$$
\begin{aligned}
& \varphi^{\prime \prime}\left(v_{k}\right)=0, \varphi^{\prime \prime \prime}\left(v_{k}\right)=3 \xi(\text { vstep })^{-2}\left(R^{T} R\right) \varphi\left(v_{k}\right), \\
& \psi^{\prime \prime}\left(v_{k}\right)=0, \psi^{\prime \prime \prime}\left(v_{k}\right)=3 \xi(\text { vstep })^{-2}\left(R^{T} R\right) \psi\left(v_{k}\right),
\end{aligned}
$$

are in effect. The formulas

$$
\begin{gathered}
x(\cdot, k)=\left(\operatorname{Id}+\xi \operatorname{vstep}\left(R^{T} R\right)\right)^{-1}(X+v \operatorname{vstep} U), \\
y(\cdot, k)=\left(\operatorname{Id}+\xi \operatorname{vstep}\left(R^{T} R\right)\right)^{-1}(Y+v \operatorname{step} V)
\end{gathered}
$$

result — in other words $x(\cdot, k)$ and $y(\cdot, k)$ are mollified versions of $X$ vstep $U$ and $Y+$ vstep $V$, respectively.

Last step. End. 
8.4. As a matter of fact, the above process simulates the partial differential system

$$
\begin{aligned}
\frac{\partial}{\partial v}\left[\begin{array}{l}
x \\
y
\end{array}\right] & =\frac{1}{f}\left[\begin{array}{cc}
0 & -1 \\
1 & 0
\end{array}\right] \frac{\partial}{\partial u}\left[\begin{array}{l}
x \\
y
\end{array}\right]-\xi^{4} \frac{\partial^{4}}{\partial u^{4}}\left[\begin{array}{l}
x \\
y
\end{array}\right], \\
f & =P\left(\nu, n(x, y) \sqrt{x_{u}^{2}+y_{u}^{2}}\right) \operatorname{sgn} \kappa(u),
\end{aligned}
$$

where the modified, and extra, terms protect against overflows and instability. The methods based on either artificial viscosity or quasi-reversibility share basic features: they all suggest perturbing the underlying partial differential equation or system in a way or another, in order to palliate obstructions. The quasi-reversibility method was introduced in [119], and improved in [151], [77]; other references are [10], [11], [27]-[28], [53], [66], [78], [90], [91], [104], [118], [167], [196]-[197], [214].

\section{Example}

For simplicity, suppose refractive index $n$ is 1 . Consider the curve - known as Tschirnhausen's cubic or trisectrix of Catalan [128], [195] — whose parametric equations read

$$
x=\frac{1}{2}\left(1-3 t^{2}\right), \quad y=\frac{t}{2}\left(3-t^{2}\right), \quad(t=\text { parameter }),
$$

and imply

$$
\text { arc length }=\frac{t}{2}\left(t^{2}+3\right), \quad t=2 \sinh \left(\frac{1}{3} \operatorname{arcsinh}(\operatorname{arclength})\right) .
$$

A geometric optical eikonal $w$ making Tschirnhausen's cubic a caustic is represented by the equations

$$
\begin{gathered}
x=\frac{1}{2}\left(1-3 t^{2}\right)-\frac{2 s t}{1+t^{2}}, \quad y=\frac{t}{2}\left(3-t^{2}\right)+\frac{s\left(1-t^{2}\right)}{1+t^{2}}, \\
w=s+\frac{t}{2}\left(3+t^{2}\right) \quad(s, t=\text { parameters })
\end{gathered}
$$

in the light region. As arguments from Section 3 show, the same eikonal can be continued in the shadow region via the equations

$$
\begin{aligned}
& x=\frac{1-2\left(s^{2}+t^{2}\right)+s^{4}-2 s^{2} t^{2}-3 t^{4}}{2\left(1+s^{2}+t^{2}\right)}, \quad y=\frac{t\left[3-2\left(s^{2}-t^{2}\right)-\left(s^{2}+t^{2}\right)^{2}\right]}{2\left(1+s^{2}+t^{2}\right)}, \\
& w=i s+t-\frac{2(i s+t)}{1+(i s+t)^{2}} x+\frac{1-(i s+t)^{2}}{1+(i s+t)^{2}} y \quad(s, t=\text { real parameters). }
\end{aligned}
$$


R. Magnanini and G. Talenti

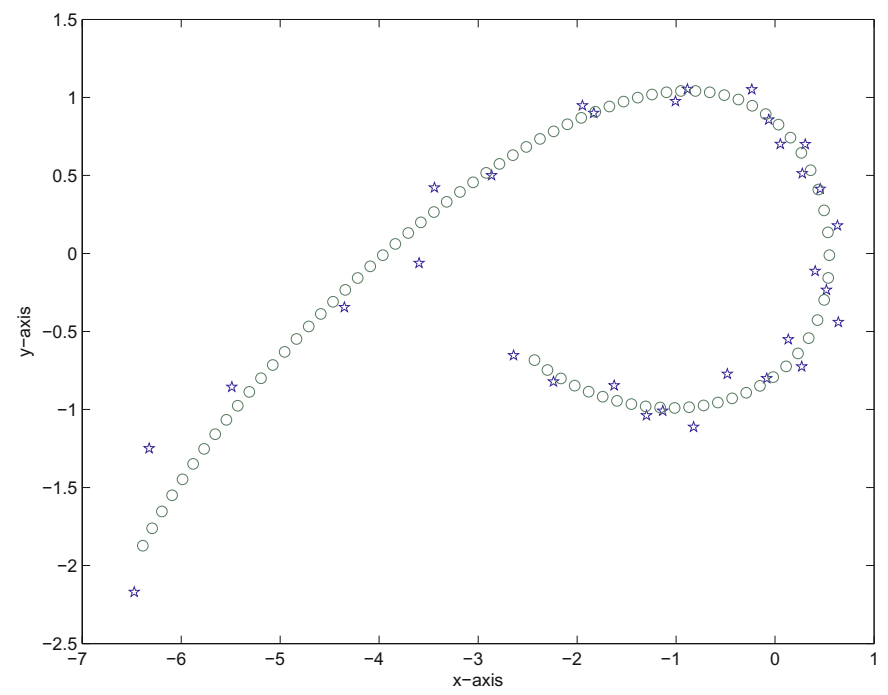

Figure 13. An arc of Tschirnhausen's cubic: a gross sampling (stars) and a denoised version (cicles).

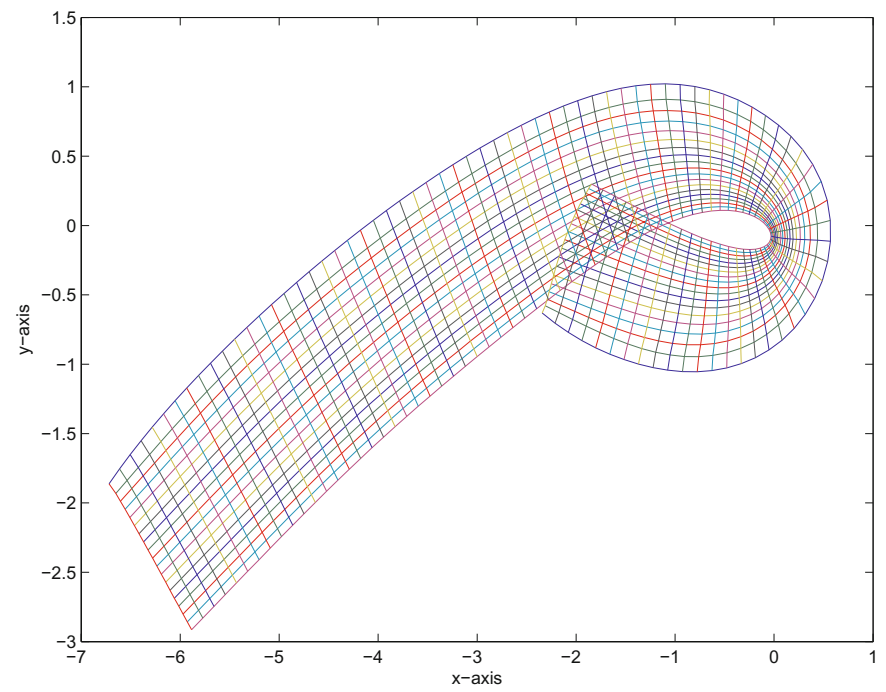

Figure 14. Level lines where either $u=$ constant or $v=$ constant.

The method from Sections 4 to 8 goes in the following way. 
On Complex-valued 2D Eikonals IV

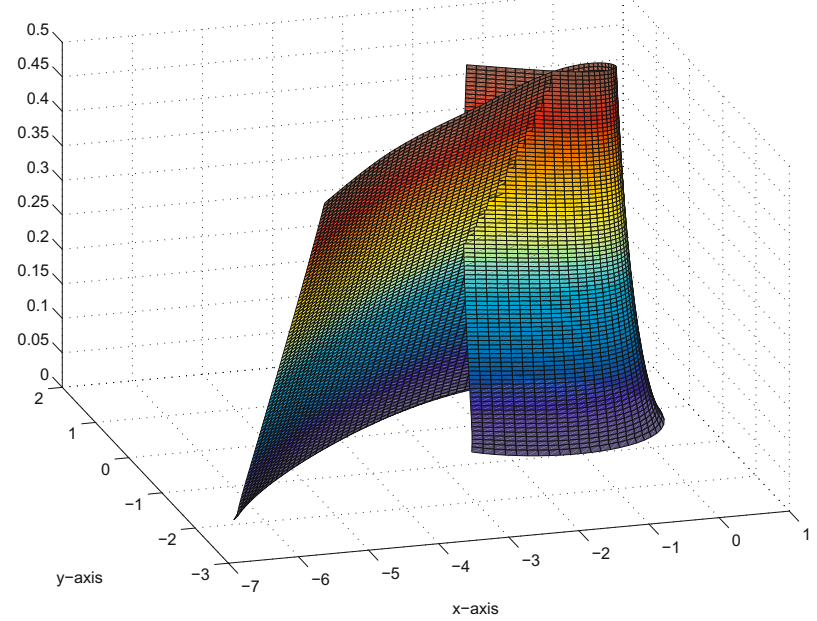

Figure 15. Plot of imaginary part $v$ versus $x$ and $y$.

(i) Consider the arc of the Tschirnhausen's cubic where

$$
-1.5 \leq t \leq 2.2
$$

for instance, and let

$$
\left(\alpha_{j}, \beta_{j}\right) \quad(j=1, \ldots, 31)
$$

be a gross sampling of such an arc — in other words,

$$
\begin{aligned}
t_{j} & =-1.5+3.7 \frac{j-1}{30} \quad(j=1, \ldots, 31), \\
\alpha_{j} & =\frac{1}{2}\left(1-3 t_{j}^{2}\right)+(5 \% \text { random noise }), \\
\beta_{j} & =\frac{t_{j}}{2}\left(3-t_{j}^{2}\right)+(5 \% \text { random noise }) .
\end{aligned}
$$

(ii) Plug gross data into the denoising process described in Section 7, i.e.

$$
\begin{gathered}
\lambda=0.005, \quad \mu=0.1260, \\
\sum_{j=1}^{31}\left[\alpha\left(\frac{j-1}{30}\right)-\alpha_{j}\right]^{2}+\lambda \int_{-\infty}^{\infty}\left[\mu^{7}\left(\alpha^{\prime \prime \prime \prime}\right)^{2}+\mu^{-1} \alpha^{2}\right] d t=\text { minimum, } \\
\sum_{j=1}^{31}\left[\beta\left(\frac{j-1}{30}\right)-\beta_{j}\right]^{2}+\lambda \int_{-\infty}^{\infty}\left[\mu^{7}\left(\beta^{\prime \prime \prime \prime}\right)^{2}+\mu^{-1} \beta^{2}\right] d t=\text { minimum, }
\end{gathered}
$$




\section{R. Magnanini and G. Talenti}

and let the path that is represented by the equations

$$
x=\alpha(t), \quad y=\beta(t), \quad 0 \leq t \leq 1,
$$

surrogate the original Tschirnhausen's cubic.

(iii) Adjust parametric equations as follows:

$$
\begin{gathered}
\frac{d t}{d u}=\left[\alpha^{\prime}(t)^{2}+\beta^{\prime}(t)^{2}\right]^{-1 / 2}, t(0)=0, \\
x=\alpha(t(u)), \quad y=\beta(t(u)), \quad 0 \leq u \leq \text { Length. }
\end{gathered}
$$

so as the working parameter become a travel time - a Runge-Kutta method fits well here.

(iv) Select requisite parameters by

$$
M=101, \quad N=91, \quad \text { vstep }=0.005, \quad \nu=0.5, \quad \xi=0.9 ;
$$

and then set the algorithm from Section 8 to work.

Results are shown in Figures 13, 14 and 15, and comfortably agree with those drawn from closed formulas.

\section{Acknowledgement}

The Authors wish to thank L. Sgheri and G.A. Viano for their valuable help and advice.

\section{Appendix}

Basic mathematical lineaments of two-dimensional geometrical optics are outlined in the next paragraphs. Selected references on geometrical optics, and on some of its generalizations and applications, are [14], [56], [80], [98], [99], [100], [105]-[106], [107], [116], [137], [144], [145], [184].

\section{A.1. Terminology}

Let $n$ be a refractive index - i.e. a tractable function of two real variables $x$ and $y$, which takes positive values only and is bounded away from zero locally. Any real-valued, suitably smooth solution $w$ to (1.1) is a geometric optical eikonal (GOE). The domain of $w$, plus parts of the relevant boundary, is a light region; the complement of it is a shadow region. The trajectories of $\nabla w$, namely the orbits of the dynamical system

$$
\left|\begin{array}{cc}
d x & d y \\
w_{x}(x, y) & w_{y}(x, y)
\end{array}\right|=0
$$


are called lines of steepest descent - irrespective of whether they are genuine lines or not. A line of steepest descent is a ray if $w$ is twice continuously differentiable in some neighborhood of it; any line of steepest descent, which is not a ray, is a caustic. (Rays are smooth curves, which have one degree of freedom and travel all over areas without intersecting one another. Caustics are exceptions in a sense: loosely speaking, they can be thought of as envelopes of rays.) The Riemannian arc length, whose element takes the form

$$
n(x, y) \sqrt{d x^{2}+d y^{2}},
$$

is known as travel time - travel time is an alias of the customary arc length in the case where $n \equiv 1$.

\section{A.2. GOEs and travel time}

(i) The restriction of any GOE $w$ to either an appertaining ray or caustic automatically coincides with a properly rescaled travel time $t$.

(ii) Let nodal line be an alias of locus of zeros. The value of any GOE $w$ at any point $(x, y)$ equals either the travel time between $(x, y)$ and a nodal line of $w$ or the negative of such a travel time - provided $(x, y)$ is not a long way off.

Proof of (i). By definition, both rays and caustics of $w$ obey

$$
d x: w_{x}(x, y)=d y: w_{y}(x, y)
$$

the equation

$$
t=\text { a rescaled travel time }
$$

is an alias of

$$
d t= \pm n(x, y) \sqrt{(d x)^{2}+(d y)^{2}} .
$$

Consequently,

$$
(d w / d t)^{2}=n^{-2}(x, y)\left(w_{x}^{2}+w_{y}^{2}\right)
$$

along any ray or caustic in question. Property (i) follows.

Property (ii) is a consequence of (i) and Fermat's principle below. 


\section{R. Magnanini and G. Talenti}

\section{A.3. Fermat's principle}

The travel time geodesics, i.e. those curves which render

$$
\int n(x, y) \sqrt{d x^{2}+d y^{2}}
$$

either a minimum or stationary, are characterized by the following secondorder ordinary differential equation:

$$
\text { curvature }=\langle\text { unit normal, } \nabla \log n(x, y)\rangle
$$

- they have geodesic curvature 0 , and are perfect straight lines in the case where $n \equiv 1$. The rays of any GOE are geodesics with respect to travel time. The travel time geodesics that are trajectories of some proper vector field - i.e. have one degree of freedom and are free from mutual intersections - are the rays of some GOE.

The foregoing statements rest upon first principles of calculus of variations, differential geometry and ordinary differential equations. Recall that the following formulas apply to any smooth parametric curve:

$$
\begin{gathered}
\text { velocity }=\sqrt{(d x / d t)^{2}+(d y / d t)^{2}}, \\
\text { unit tangent }=(\text { velocity })^{-1} \frac{d}{d t}\left[\begin{array}{l}
x \\
y
\end{array}\right], \\
\text { unit normal }=\left[\begin{array}{cc}
0 & -1 \\
1 & 0
\end{array}\right] \text { (unit tangent) }
\end{gathered}
$$

$$
\begin{gathered}
(\text { velocity })^{-1} \frac{d}{d t}(\text { unit tangent })=(\text { curvature }) \times(\text { unit normal }), \\
(\text { velocity })^{-1} \frac{d}{d t}(\text { unit normal })=-(\text { curvature }) \times(\text { unit tangent }), \\
\text { curvature }=(\text { velocity })^{-3}\left|\begin{array}{cc}
d x / d t & d^{2} x / d t^{2} \\
d y / d t & d^{2} y / d t
\end{array}\right| ; \\
\text { curvature }=(\text { velocity })^{-3} h^{-3}\left|\begin{array}{ccc}
x(t-h) & y(t-h) & 1 \\
x(t) & y(t) & 1 \\
x(t+h) & y(t+h) & 1
\end{array}\right|+O\left(h^{2}\right),
\end{gathered}
$$




$$
\begin{aligned}
& \text { curvature }=(\text { velocity })^{-3} h^{-3}\left\{\left|\begin{array}{ccc}
x(t) & y(t) & 1 \\
x(t+h) & y(t+h) & 1 \\
x(t+2 h) & y(t+2 h) & 1
\end{array}\right|\right. \\
&\left.-\frac{1}{2}\left|\begin{array}{ccc}
x(t+h) & y(t+h) & 1 \\
x(t+2 h) & y(t+2 h) & 1 \\
x(t+3 h) & y(t+3 h) & 1
\end{array}\right|\right\}+O\left(h^{2}\right) .
\end{aligned}
$$

Recall that the formula

$$
\begin{aligned}
& \text { curvature of the lines of steepest descent } \\
& =\text { Jacobian determinant of }|\nabla w|^{-1} \& w
\end{aligned}
$$

applies whenever $w$ is smooth and has no critical point. Recall also that

$$
\begin{gathered}
n(x, y) \times(\text { Geodesic curvature }) \\
=\text { Euclidean curvature }-\langle\text { unit normal, } \nabla \log n(x, y)\rangle
\end{gathered}
$$

if travel time is an alternative Riemannian metric in force.

\section{A.4. Initial value problems and geometry of their solutions}

The condition of taking given values along some given path is qualified initial according to usage. Seeking a GOE, which obeys some initial condition, is an initial value problem. Such a problem has either two different solutions or no solution at all, depending on whether the eikonal equation and the initial condition match or conflict. Generally speaking, a solution $w$ can be detected in the former case by successively detecting the objects listed below, based upon the arguments provided.

- The values of $\nabla w$ along the initial curve.

Since the eikonal equation specifies the length of $\nabla w$ and the initial condition identifies a tangential component of $\nabla w$, the normal component of $\nabla w$ along the initial curve comes out in two different modes.

- The rays of $w$.

An ODE reasoning demonstrates that the travel time geodesics, which live near the initial curve and leave it with the same direction as $\nabla w$, are the trajectories of a smooth vector field. By Fermat's principle, these geodesics are the requested rays indeed.

- The values of $w$ itself on each ray.

Property (i) from Section 2 fits the situation well. 


\section{R. Magnanini and G. Talenti}

\section{A.5. Standard initial value problems}

The present item concerns existence, regularity and the number of GOEs that satisfy orthodox initial conditions.

Assume henceforth all ingredients are smooth and let IC stand for initial curve in shorthand. Let

$$
x=\alpha(t), \quad y=\beta(t), \quad a \leq t \leq b
$$

be a parametric representation of IC such that

$$
t=\text { a travel time. }
$$

Let the initial condition imply

$$
w(x, y)=\gamma(t)
$$

as $x, y$ and $t$ satisfy (A.1), and assume

$$
\left|\frac{d \gamma}{d t}(t)\right|<1
$$

for $a \leq t \leq b$. Then exactly two GOEs satisfy the initial condition displayed. Moreover, these eikonals are smooth in a full neighborhood of IC - the relevant light regions surround it completely.

The case where refractive index $n$ is constant involves explicit formulas, as well as gives evidence to interactions among the eikonal equation, Burgers-type equations and Bäcklund transformations. Assume

$$
n \equiv 1
$$

and let (A.1) to (A.3) be in force. Define $\varphi, \psi, \omega, p, q$ by

$$
\begin{gathered}
\cos \varphi=\alpha^{\prime}, \quad \sin \varphi=\beta^{\prime} ; \\
\cos \psi=\gamma^{\prime}, \quad \sin \psi= \pm \sqrt{1-\left(\gamma^{\prime}\right)^{2}} ; \\
\omega=\varphi+\psi ; \\
p=\cos \omega, \quad q=\sin \omega .
\end{gathered}
$$

Since the appurtenant Jacobian matrix equals

$$
\left[\begin{array}{cc}
q(s) & p(s) \\
-p(s) & q(s)
\end{array}\right]\left[\begin{array}{cc}
\sin \psi(s)-(w-\gamma(s)) d \omega(s) / d s & 0 \\
0 & 1
\end{array}\right]
$$

the pair

$$
\left[\begin{array}{l}
x \\
y
\end{array}\right]=\left[\begin{array}{l}
\alpha(s) \\
\beta(s)
\end{array}\right]+(w-\gamma(s))\left[\begin{array}{c}
p(s) \\
q(s)
\end{array}\right]
$$


makes $s$ and $w$ implicit functions of $x$ and $y$ in a neighborhood of IC. The properties listed below ensue. Function $s$ obeys the equations

$$
\begin{gathered}
-q(s)(x-\alpha(s))+p(s)(y-\beta(s))=0 \\
p(s) \frac{\partial s}{\partial x}+q(s) \frac{\partial s}{\partial y}=0 \\
\left(\frac{\partial s}{\partial x}\right)^{2}+\left(\frac{\partial s}{\partial y}\right)^{2}=\left[\sin \psi(s)-(w-\gamma(s)) \frac{d \omega(s)}{d s}\right]^{-2} .
\end{gathered}
$$

(The first assures that the level lines of $s$ are straight, the second is a PDE of Burgers type.) Function $w$ obeys the following eikonal equation:

$$
\left(\frac{\partial w}{\partial x}\right)^{2}+\left(\frac{\partial w}{\partial y}\right)^{2}=1
$$

Functions $s$ and $w$ are related by the equations

$$
\begin{gathered}
\nabla w=\left[\begin{array}{c}
p(s) \\
q(s)
\end{array}\right] \\
{\left[\begin{array}{ll}
w_{x x} & w_{x y} \\
w_{x y} & w_{y y}
\end{array}\right]=\frac{d \omega(s)}{d s}\left[\begin{array}{c}
-w_{y} \\
w_{x}
\end{array}\right]\left[\begin{array}{ll}
s_{x} & s_{y}
\end{array}\right] .}
\end{gathered}
$$

(The former is a Bäcklund transformation, which pairs solutions to the Burgers and the eikonal equations mentioned above. It assures that $\nabla s$ and $\nabla w$ are orthogonal, and the level lines of $s$ are both lines of steepest descent and isoclines of $w$.) The Euclidean metric obeys

$$
d x^{2}+d y^{2}=|\nabla s|^{-2} d s^{2}+d w^{2} .
$$

There holds

$$
s(x, y)=t, \quad w(x, y)=\gamma(t)
$$

as $x, y$ and $t$ satisfy (A.1). If $\omega$ is free from critical points, the line where

$$
\left[\begin{array}{l}
x \\
y
\end{array}\right]=\left[\begin{array}{c}
\alpha(s) \\
\beta(s)
\end{array}\right]+\frac{\sin \psi(s)}{d \omega(s) / d s}\left[\begin{array}{c}
p(s) \\
q(s)
\end{array}\right]
$$

is a caustic. (Such a line is an envelope of the level lines of $s$. Function $s$ develops shocks along it, the restriction of $w$ to it equals arc length, and the second-order derivatives of $w$ blow up there.) 


\section{R. Magnanini and G. Talenti}

\section{A.6. Borderline initial value problems and caustics}

The present item is a recipe for producing caustics, which involves coupling the eikonal equation with borderline initial conditions. In the case where refractive index $n$ is identically 1 , any smooth convex curve can be viewed as a caustic provided a GOE is detected, whose restriction to the curve in hand equals a relevant arc length.

Let (A.1) specify IC and let (A.2) hold. Let the initial condition imply

$$
w(x, y)=t
$$

as $x, y$ and $t$ satisfy (A.1). Assume that the appropriate geodesic curvature of IC is free from zeros - in other words, let (A.1) and the equation

$$
\kappa(\text { velocity })^{2}=\text { Euclidean curvature }-\langle\text { unit normal, } \nabla \log n(x, y)\rangle
$$

result in

$$
\kappa \text { vanishes nowhere. }
$$

Then exactly two GOEs satisfy the present initial condition. Both these eikonals fail to exist on both sides of, and be smooth near IC. They turn IC into a caustic, and make the side of it, which

$$
(\operatorname{sgn} \kappa) \times(\text { unit normal })
$$

points to, a shadow region. Either eikonal in hand obeys

$w(x, y)=s \pm \frac{2 \sqrt{2}}{3}|r|^{\frac{3}{2}}|\kappa(s)|^{\frac{1}{2}}+O\left(r^{2}\right), \quad \Delta w(x, y)= \pm \frac{1}{\sqrt{2}}|r|^{-\frac{1}{2}}|\kappa(s)|^{\frac{1}{2}}+O(1)$

at every point $(x, y)$ that belongs to the light region and is close enough to IC - in particular, the two-sheeted surface, made up of the two eikonals in hand, exhibits an edge of regression above IC.

Here $r$ and $s$ are the curvilinear coordinates that the pair

$$
\left[\begin{array}{l}
x \\
y
\end{array}\right]=\left[\begin{array}{c}
\alpha(s) \\
\beta(s)
\end{array}\right]+r\left(\alpha^{\prime}(s)^{2}+\beta^{\prime}(s)^{2}\right)^{-\frac{1}{2}}\left[\begin{array}{c}
-\beta^{\prime}(s) \\
\alpha^{\prime}(s)
\end{array}\right]
$$

relates to rectilinear coordinates $x$ and $y$. Coordinate $r$ is a signed distance from IC, coordinate $s$ makes $(\alpha(s(x, y)), \beta(s(x, y)))$ the orthogonal projection of $(x, y)$ on IC. The former is constant on the parallel lines to IC and obeys the eikonal equation

$$
\left(\frac{\partial r}{\partial x}\right)^{2}+\left(\frac{\partial r}{\partial y}\right)^{2}=1
$$


the latter is constant on the normal straight-lines to IC and obeys the following Burgers-type equation:

$$
\left|\begin{array}{cc}
\partial s / \partial x & \partial s / \partial y \\
\alpha^{\prime}(s) & \beta^{\prime}(s)
\end{array}\right|=0
$$

Both are subject to the Bäcklund transformation

$$
\nabla r=\left[\alpha^{\prime}(s)^{2}+\beta^{\prime}(s)^{2}\right]^{-\frac{1}{2}}\left[\begin{array}{c}
-\beta^{\prime}(s) \\
\alpha^{\prime}(s)
\end{array}\right]
$$

and exhibit singularities along the evolute of IC.

\section{References}

[1] A. Abdukarimov, A problem of analytic continuation from a discrete set for functions of complex variables. Approximate solution methods and questions of the well-posedness of inverse problems, 5-8, Vychisl. Tsentr, Novosibirsk, 1981.

[2] S. Ahn \& U.J. Choi \& A.G. Ramm, A scheme for stable numerical differentiation. J. Comput. Appl. Math. 186 (2006) 325-334.

[3] G. Alessandrini, On differentiation of approximately given functions. Applicable Analysis 11 (1980) 45-59.

[4] G. Alessandrini, An extrapolation problem for harmonic functions. Boll. UMI 17B (1980) 860-875.

[5] O.M. Alifanov, Methods of solving ill-posed problems. Translated from the Russian original in J. Engrg. Phys. 45 (1984) 1237-1245.

[6] K.A. Ames \& V. Isakov, An explicit stability estimate for an ill-posed Cauchy problem for the wave equation. J. Math. Anal. Appl. 156 (1991) 597-610.

[7] R. S. Anderssen \& P. Bloomfield, Numerical differentiation procedures for non-exact data. Numer. Math. 22 (1973/74) 157-182.

[8] R.S. Anderssen \& P. Bloomfield, A time series approach to numerical differentiation. Technometrics 16 (1974) 69-75.

[9] R.S. Anderssen \& F.R. de Hoog, Finite difference methods for the numerical differentiation of non-exact data. Computing 33 (1984) 259-267.

[10] D.D. Ang \& D.D. Trong, A Cauchy problem for elliptic equations: quasireversibility and error estimates. Vietnam J. Math. 32 (2004), Special Issue, $9-17$.

[11] D.D. Ang \& D.D. Trong \& M. Yamamoto, A Cauchy like problem in plane elasticity:regularization by quasi-reversibility with error estimates. Vietnam J. Math. 32 (2004), no.2, 197-208. 


\section{R. Magnanini and G. Talenti}

[12] M.M. Aripov \& M. Khaidarov, A Cauchy problem for a nonlinear heat equation in an inhomogeneous medium (in Russian). Dokl. Akad. Nauk SSSR 2 (1986) 11-13.

[13] M.I. Baart, Computational experience with the spectral smoothing method for differentiating noisy data. J. Comput. Phys. 42 (1981) 141-151.

[14] V.M. Babich \& V.S. Buldyrev, Short-wavelength diffraction theory. SpringerVerlag, 1991.

[15] A.B. Bakushinskii \& A.V. Goncharskii, Ill-posed problems: theory and applications. Kluwer, 1995.

[16] A.B. Bakushinskii \& M.Yu. Kokurin \& S.K. Paymerov, On error estimates of difference solution method for ill-posed Cauchy problems in a Hilbert space. J. Inverse Ill-Posed Problems 16 (2008) 553-565.

[17] J.B. Bell, The noncharacteristic Cauchy problem for a class of equations with time dependence. I: problems in one space dimension. II: multidimensional problems. SIAM J. Math. Anal. 12 (1981), pages 759-777 and 778-797.

[18] F. Berntsson \& L. Eldén, Numerical solution of a Cauchy problem for the Laplace equation. Inverse Problems 17 (2001) 839-854.

[19] M. Bertero, Regularization methods for linear inverse problems. In: Inverse problems (G. Talenti editor), Lecture Notes in Math. vol. 1225, Springer 1986.

[20] M. Bertero, Linear inverse and ill-posed problems. Advances in Electronics and Electron Physics 75 (1989) 2-120.

[21] M. Bertero, The use of a priori information in the solution of ill-posed problems. Pages 19-27 in Partial differential equations and applications (P. Marcellini \& G. Talenti \& E. Vesentini editors), Lecture Notes in Pure and Appl. Math. 177, Dekker, 1996.

[22] M. Bertero \& C. DeMol \& G.A. Viano, The stability of inverse problems. Pages 161-214 in Inverse Scattering Problems in Optics (H. Baltes editor), Topics in Current Physics, vol.20, Springer, 1980.

[23] M. Bertero \& G.A. Viano, On the numerical analytic continuation of the proton electromagnetic form factors. Nuovo Cimento 39 (1965) 1915-1920.

[24] M. Bertero \& G.A. Viano, On probabilistic methods for the solution of improperly posed problems. Boll. UMI B (5) 15 (1978) 483-508.

[25] N. Bogdanova \& T. Kupenova, A numerical method for analytic continuation of holomorphic functions outside the real axis. Godishnik Vyss. Ucebn. Zaved. Tekh. Fiz. 16 (1979) 91-96.

[26] D. Bouche \& F. Molinet, Méthodes asymptotiques en Électromagnètisme. Springer-Verlag, 1994. 
[27] L. Bourgeois, A mixed formulation of quasi-reversibility to solve the Cauchy problem for Laplace's equation. Inverse Problems 21 (2005) 1087-1104.

[28] L. Bourgeois, Convergence rates for the quasi-reversibility method to solve the Cauchy problem for Laplace's equation. Inverse Problems 22 (2006) 413430.

[29] N. Boussetila \& F. Rebbani, Optimal regularization methods for ill-posed problems. Electron. J. Differential Equations 147 (2006) 15 pp.

[30] A. Bressan, An ill-posed Cauchy problem for a hyperbolic system in two space dimensions. Rend. Sem. Mat. Univ. Padova 110 (2003) 103-117.

[31] W.L. Briggs \& V.E. Henson, The DFT. SIAM, 1995.

[32] J. Cannon, A Cauchy problem for the heat equation. Ann. Mat. Pura Appl. 66 (1964) 155-165.

[33] J. Cannon \& J. Douglas, The Cauchy problem for the heat equation. SIAM J. Numer. Anal. 4 (1967) 317-336.

[34] J. Cannon \& R.E. Ewing, A direct numerical procedure for the Cauchy problem for the heat equation. J. Math. Anal. Appl. 56 (1976) 7-17.

[35] J.R. Cannon \& K. Miller, Some problems in numerical analytic continuation. SIAM J. Numer. Anal. 2 (1965) 87-98.

[36] A.S. Carasso, A stable marching scheme for an ill-posed initial value problem. Pages 11-35 in Improperly posed problems and their numerical treatment (Oberwolfach, 1982), Internat. Schriftenreihe Numer. Math. 63, Birkhuser, 1983.

[37] S.J. Chapman \& J.M.H. Lawry \& J.R. Ockendon \& R.H. Tew, On the theory of complex rays. SIAM Review 41 (1999) 417-509.

[38] J. Cheng \& X.Z. Jia \& Y.B. Wang, Numerical differentiation and its applications. Inverse Probl. Sci. Eng. 15 (2007) 339-357.

[39] S. Choudhary \& L.B. Felsen, Asymptotic theory for inhomogeneous waves, IEEE, Transactions on Antennas and Propagation 21 (1973) 827-842.

[40] S. Choudhary \& L.B. Felsen, Analysis of Gaussian beam propagation and diffraction by inhomogeneous wave tracking. Proceedings of IEEE 62 (1974) 1530,1541 .

[41] S. Ciulli \& T.D. Spearman, Analytic continuation from data points with unequal errors. J. Math. Phys. 23 (1982) 1752-1764.

[42] S. Ciulli \& T.D. Spearman, Analytic continuation from empirical data: a direct approach to the stabilization problem. J. Math. Phys. 37 (1996) 933941.

[43] D. Colton, The noncharacteristic Cauchy problem for parabolic equations in two space variables. Proc. Amer. Math. Soc. 41 (1973) 551-556. 


\section{R. Magnanini and G. Talenti}

[44] D.D. Cox, Asymptotics of M-type smoothing splines. Ann. Statist. 11 (1983) $530-551$.

[45] F. Cucker \& S. Smale, On the mathematical foundations of learning. Bull. Amer. Math. Soc. 39 (2002) 1-49.

[46] L.A. Čudov, Difference schemes and ill-posed problems for partial differential equations (in Russian). Pages 34-62 in Computing methods and programming 8, Izdat. Moskow Univ., 1967.

[47] J. Cullum, Numerical differentiation and regularization. SIAM J. Numer. Anal. 8b(1975) 254-265.

[48] A.R. Davies, Optimality in numerical differentiation. Proc. Centre Math. Anal., Austral. Nat. Univ., Canberra, 1988.

[49] V.N. Denisov, Stabilization of the solution of the Cauchy problem for the heat equation. Translated from the Russian original in Soviet Math. Dokl. 37 (1988) 688-692.

[50] Dinh Nho Hao \& H. Sahli, Stable analytic continuation by mollification and the fast Fourier transform. Methods of complex and Clifford analysis, 143152, SAS int. Publ., Delhi, 2004.

[51] T.F. Dolgopolova, Finite-dimensional regularization in numerical differentiation of periodic functions. Ural. Gos. Univ. Mat. Zap. 7 (1969/70) 27-33.

[52] T.F. Dolgopolova \& V.K. Ivanov, On numerical differentiation. USSR Computational Math. and Math. Phys. 6 (1966) 223-232.

[53] J.R. Dorroh \& X. Ru, The application of the method of quasi-reversibility to the sideways heat equation. J. Math. Anal. Appl. 236 (1999) 503-519.

[54] J. Douglas, A numerical method for analytic continuation. Boundary problems in differential equations, pp. 179-189, University of Wisconsin Press, 1960.

[55] J. Douglas, Approximate continuation of harmonic and parabolic functions. Pages 353-364 in Numerical solution of partial differential equations (Proc. Symp. Univ. Maryland, 1965), Academic Press, 1966.

[56] J.J. Duistermaat, Oscillatory integrals, Lagrange immersions and unfolding of singularities. Comm. Pure Appl. Math. 27 (1974) 207-281.

[57] R.A. Egorchenkov \& Yu.A. Kravtsov, Numerical implementation of complex geometrical optics. Radiophys. and Quantum Electronics 43 (2000), no.7, 569-575 (2001).

[58] R.A. Egorchenkov \& Yu.A. Kravtsov, Diffraction of super-Gaussian beams as described by the complex geometrical optics. Radiophys. and Quantum Electronics 43 (2000), no.10, 798-804 (2001).

[59] Yu.V. Egorov \& V.A. Kondratiev, On a problem of numerical differentiation. Moscow Univ. Math. Bull. 44 (1989) 85-87. 


\section{On Complex-valued 2D Eikonals IV}

[60] P. Einzinger \& L.B. Felsen, Evanescent waves and complex rays. IEEE, Transactions on Antennas and Propagation AP30 (1982) 594-605.

[61] P. Einzinger \& S. Raz, On the asymptotic theory of inhomogeneous wave tracking. Radio Science 15 (1980) 763-771.

[62] L. Eldén, Approximations for a Cauchy problem for the heat equation. Inverse Problems 3 (1987) 263-273.

[63] L. Eldén, Hyperbolic approximations for a Cauchy problem for the heat equation. Inverse Problems 4 (1988) 59-70.

[64] L.C. Evans, Partial differential equations. Amer. Math. Soc., 1998.

[65] T. Evgeniou \& M. Pontil \& T. Poggio, Regularization networks and support vector machines. Advances in Computational Math. 13 (2000) 1-50.

[66] R.E. Ewing, The approximation of certain parabolic equations backward in time by Sobolev equations. SIAM J. Math. Anal. 6 (1975) 91-95.

[67] R.E. Ewing, The Cauchy problem for a linear parabolic differential equation. J. Math. Anal. Appl. 71 (1979) 167-186.

[68] R.E. Ewing \& R.S. Falk, Numerical approximation of a Cauchy problem for a parabolic partial differential equation. Math. Comp. 33(1979) 1125-1144.

[69] A.M. Fedotov, Theoretical justification of computational algorithms for problems of analytic continuation (in Russian). Siberian Math. J. 33 (1992) 511519 .

[70] A.M. Fedotov, Analytic continuation of functions from discrete sets. J. Inverse Ill-Posed Probl. 2 (1994) 235-252.

[71] L.B. Felsen, Complex-source-point solutions of the field equation and their relation to the propagation and scattering of Gaussian beams. Symposia Mathematica 18 (1976) 39-56.

[72] L.B. Felsen, Evanescent waves, J. Opt. Soc. Am. 66 (1976) 751-760.

[73] J.N. Franklin, On Tikhonov method for ill-posed problems. Math. Computation 28 (1974) 889-907.

[74] J.N. Franklin, Analytic continuation by the fast Fourier transform. SIAM J. Statistic. Comput. 11 (1990) 112-122.

[75] H. Fujiwara \& H. Imai \& T. Takeuchi \& Y. Iso, Numerical treatment of analytic continuation with multiple-precision arithmetic. Hokkaido Math. J. 36 (2007) 837-847.

[76] A.A. Fuki \& Yu.A. Kravtsov \& O.N. Naida, Geometrical optics of weakly anisotropic media. Gordon and Breach Science Publishers, 1998.

[77] H. Gajewski \& K. Zacharias, Regularizing a class of ill-posed problems for evolution equations (German). J. Math. Anal. Appl. 38 (1972) 784-789. 
[78] J.A. Goldstein, Uniqueness in nonlinear Cauchy problems in Banach spaces Proc. Amer. Math. Soc. 53 (1975) 91-95.

[79] C. W. Groetsch, Differentiation of approximately specified functions. Amer Math. Monthly 98 (1991) 847-850.

[80] V. Guillemin \& S. Sternsberg, Geometric asymptotics. Amer. Math. Soc 1977.

[81] K.N. Gurjanova, A method of numerical analytic continuation (in Russian)' Izv. Vyss. Ucebn. Zaved Matematika 1966 (1966) 47-55.

[82] J. Hadamard, Sur les problmes aux dérivées partielles et leur significatio: physique (in French). Bull. Univ. Princeton 13 (1902) 49-52.

[83] J. Hadamard, Lectures on Cauchy's problem in linear partial differentis equations. Yale University Press, 1923.

[84] G. Hammerlin \& K.H. Hoffman (editors), Improperly posed problems an their numerical treatment. Birkhuser, 1983.

[85] H. Han \& H.J. Reinhardt, Some stability estimates for Cauchy problems fo elliptic equations. J. Inv. Ill-Posed Problems 5 (1997) 437-454.

[86] P.C. Hansen, Rank-deficient and discrete ill-posed problems. SIAM 1998.

[87] E. Heyman \& L.B. Felsen, Evanescent waves and complex rays for modo propagation in curved open waveguides. SIAM J. Appl. Math. 43 (1983 855-884.

[88] J. van der Hoeven, On effective analytic continuation. Math. Comput. Sci. (2007) 111-175.

[89] B. Hofmann, On the degree of ill-posedness for nonlinear problems. J. Invers Ill-Posed Problems 2 (1994) 61-76.

[90] Y. Huang, Modified quasi-reversibility method for final value problems i: Banach spaces. J. Math. Anal. Appl. 340 (2008) 757-769.

[91] Y. Huang \& Q. Zheng, Regularization for ill-posed Cauchy problems associ ated with generators of analytic semigroups. J. Differ. Equations 203 (2004 38-54.

[92] V. Isakov, Inverse problems for partial differential equations. Springer, $199 \varepsilon$

[93] V.K. Ivanov \& V.V. Vasin \& V.P. Tanana, Theory of linear ill-posed problem and its applications (in Russian). Izdat. Nauka, Moskow, 1978.

[94] F. John, A note on improper problems in partial differential equations Comm. Pure Appl. Math. 8 (1955) 494-495.

[95] F. John, Numerical solution of the equation of heat conduction for proceedin times. Ann. Mat. Pura Appl. 40 (1955) 129-142.

[96] F. John, Continuous dependence on data for solutions with a prescribe bound. Comm. Pure Appl. Math. 13 (1960) 551-585. 
[97] L.W. Johnson \& R.D. Riess, An error analysis for numerical differentiation. J. Inst. Math: Appl. 11 (1973) 115-120.

[98] D.S. Jones, The theory of electromagnetism. Pergamon Press, 1964.

[99] J. Keller, Rays, waves and asymptotics. Bull. Amer. Math. Soc. 84 (1978) $727-750$.

[100] J.B. Keller \& R.M. Lewis, Asymptotic methods for partial differential equations: the reduced wave equation and Maxwells equations. Plenum Press, 1995.

[101] R. Kenyon \& A. Okounkov, Limit shapes and the complex Burgers equation. Acta Math. 199 (2007) 263-302.

[102] N. Khanal \& J. Wu \& J.M. Yuan \& B.Y. Zhang, Complex-valued Burgers and KdV-Burgers equations. ArXiv:0901.2132v1 [math.AP] 14 Jan 2009.

[103] J.T. King \& D.A. Murio, Numerical differentiation by finite-dimensional regularization. IMA J. Numer. Anal. 6 (1986) 65-85.

[104] M.V. Klibanov \& F. Santosa, A computational quasi-reversibility method for Cauchy problem for Laplace's equation. SIAM J. Appl. Math. 51 (1991) $1653-1675$.

[105] M. Kline, An asymptotic solution of Maxwell equation. Comm Pure Appl. Math. 4 (1951) 225-262.

[106] M. Kline, Asymptotic solution of linear hyperbolic partial differential equations. J. Rat. Mech. Anal. 3 (1954) 315-342.

[107] M. Kline \& J.W. Kay, Electromagnetic theory and geometrical optics. Wiley, 1965.

[108] P. Knabner \& S. Vessella, Stabilization of ill-posed Cauchy problems for parabolic equations. Ann. Mat. Pura Appl. 149 (1987) 393-409.

[109] R.J. Knops, Instability and the ill-posed Cauchy problem in elasticity. Pages 357-382 in Mechanics of solids, Pergamon Press, 1982.

[110] I. Knowles \& R. Wallace, A variational method for numerical differentiation. Numer. Math. 70 (1995) 91-110.

[111] E.V. Kolpakova, Numerical solution of the problem of reconstructing the derivative (in Russian). Differencial'nye Uravnenija I Vychisl. Mat. Vyp. 6 (1976) 137-143.

[112] Yu.A. Kravtsov, A modification of the geometrical optics method (in Russian). Radiofizika 7 (1964) 664-673.

[113] Yu.A. Kravtsov, Asymptotic solutions of Maxwells equations near a caustic (in Russian). Radiofizika 7 (1964) 1049-1056.

[114] Yu.A. Kravtsov, Complex rays and complex caustics (in Russian). Radiofizika 10 (1967) 1283-1304. 


\section{R. Magnanini and G. Talenti}

[115] Yu.A. Kravtsov \& G.W: Forbes \& A.A: Asatryan, Theory and applications of complex rays. Progress in optics, vol. XXXIX, 1-62, North-Holland, 1999.

[116] Yu.A. Kravtsov \& Yu.I. Orlov, Geometrical optics of inhomogeneous media. Springer-Verlag, 1990.

[117] Yu.A. Kravtsov \& Yu.I. Orlov, Caustics, catastrophes and wave fields. Springer-Verlag, 1999.

[118] R. Lattès, Non-well-set problems and the method of quasi reversibility. Functional Analysis and Optimization pp. 99-113, Academic Press, 1966.

[119] R. Lattès \& J.L. Lions, Méthode de quasi-reversibilité et applications (in French). Dunod, 1967.

[120] M.M. Lavrentiev, On the Cauchy problem for Laplace equation (in Russian). Dokl. Akad. Nauk SSSR 102 (1952).

[121] M.M. Lavrentiev, On the Cauchy problem for Laplace equation (in Russian). Izvest. Akad. Nauk SSSR 120 (1956) 819-842.

[122] M.M. Lavrentiev, On the Cauchy problem for linear elliptic equations of second order (in Russian). Dokl. Akad. Nauk SSSR 112 (1957) 195-197.

[123] M.M. Lavrentiev, Some improperly posed problems of mathematical physics. Springer, 1967.

[124] M.M. Lavrentiev, Improperly posed problems of Mathematical Physics. Amer. Math. Soc., 1986.

[125] M.M. Lavrentiev \& B.K. Amonov, Determination of the solution of the diffusion equation from its values on discrete sets (in Russian). Dokl. Akad. Nauk SSSR 221 (1975) 1284-1285.

[126] M.M. Lavrentiev \& V.G. Romanov \& S.P. Shishatskii, Ill-posed problems of mathematical physics and analysis. Amer. Math. Soc., 1986.

[127] M.M. Lavrentiev \& V.G. Vasiliev, On the formulation of some improperly posed problems of mathematical physics (in Russian). Sibirsk Mat. . 7 (1966) 559-576.

[128] J. D. Lawrence, A catalog of special curves. Dover Publications, 1972.

[129] H.A. Levine \& S. Vessella, Estimates and regularization for solutions of some ill-posed problems of elliptic and parabolic type. Rend. Circ. Mat. Palermo 123 (1980) 161-183.

[130] R.M. Lewis, Analytic continuation using numerical methods. Pages 45-81 in: Methods in Computational Physics 4 (B. Adler \& S. Fernbach \& M. Rotenberg editors), Academic Press 1965.

[131] R.M. Lewis \& N. Bleistein \& D. Ludwig, Uniform asymptotic theory of creeping waves. Comm. Pure Appl. Math. 20 (1967) 295-328.

[132] J.L. Lions, Sur la stabilization de certaines problèmes mal posés (in French). Rend. Sem. Mat. Fis. Milano 36 (1966) 80-87. 
[133] S. Lu \& S.V. Pereverzev, Numerical differentiation from a viewpoint of regularization theory. Math. Comp. 75 (2006) 1853-1870.

[134] S. Lu \& Y.B. Wang, First and second order numerical differentiation with Tikhonov regularization. Front. Math. China 1 (2006) 354-367.

[135] D. Ludwig, Uniform asymptotic expansions at a caustic. Comm. Pure Appl. Math. 19 (1966) 215-250.

[136] D. Ludwig, Uniform asymptotic expansion of the field scattered by a convex object at high frequencies. Comm. Pure Appl. Math. 20 (1967) 103-138.

[137] R.K. Lunenburg, Mathematical theory of optics. Univ. of California Press, 1964.

[138] R. Magnanini \& G. Talenti, On complex-valued solutions to a 2-D eikonal equation. Part One: qualitative properties, Contemporary Math. 283 (1999), 203-229.

[139] R. Magnanini \& G. Talenti, On Complex-Valued Solutions to a 2D Eikonal Equation. Part Two: Existence Theorems, SIAM J. Math. Anal. 34 (2002) 805-835.

[140] R. Magnanini \& G. Talenti, On Complex-Valued Solutions to a 2D Eikonal Equation. Part Three: analysis of a Bäcklund transformation, Appl. Anal. 85, no. 1-3 (2006), 249-276.

[141] R. Magnanini \& G. Talenti, Approaching a partial differential equation of mixed elliptic-hyperbolic type. Pages 263-276 in Ill-posed and Inverse Problems (S.I. Kabanikin \& V.G. Romanov Editors), VSP, Netherlands (2002).

[142] V.P. Maslov, Ill posed Cauchy problems for ideal gas equations and their regularization. Expos 19 in quations aux drives partielles (Saint Jean de Monts, 1987), cole Polytechnique, 1987.

[143] V.P. Maslov, Resonance ill-posedness (in Russian). Pages 50-62 in Current problems in applied mathematics and in mathematical physics (Russian), Nauka, Moscow, 1988

[144] V.P. Maslov \& M.V. Fedoriuk, Semi-classical approximation in quantum mechanics. Reidel Publishing Company, 1981.

[145] V.P. Maslov \& G.A. Omel'yanov, Geometric asymptotics for nonlinear PDE. Amer. Math. Soc., 2001.

[146] G. Miel \& R. Mooney, On the condition number of Lagrangian numerical differentiation. Appl. Math. Comput. 16 (1985) 241-252.

[147] K. Miller, Three circle theorems in partial differential equations and applications to improperly posed problems. Arch. Rational Mech. Anal. 16 (1964) $126-154$.

[148] K. Miller, Least square methods for ill-posed problems with a prescribed bound. SIAM J. Math. Anal. 1 (1970) 52-74. 


\section{R. Magnanini and G. Talenti}

[149] K. Miller, Stabilized numerical analytic prolongation with poles. SIAM J. Appl. Math. 18 (1970) 346-363.

[150] K. Miller, Stabilized numerical methods for location of poles by analytic continuation. Pages 9-20 in Studies in Numerical Analysis 2, Numerical Solutions of Nonlinear Problems, SIAM 1970.

[151] K. Miller, Stabilized quasi-reversibility and other nearly-best-possible methods for non-well-posed problems. Pages 161-176 from Lecture Notes in Mathematics, vol. 316, Springer, 1973.

[152] K. Miller \& G.A. Viano, On the necessity of nearly-best-possible methods for analytic continuation of scattering data. J. Math. Phys. 14 (1973) 1037-1048.

[153] P. Monk, Error estimates for a numerical method for an ill-posed Cauchy problem for the heat equation. SIAM J. Numer. Anal. 23 (1986) 1155-1172.

[154] V.A. Morozov, Methods for solving incorrectly posed problems. Springer, 1984.

[155] D.A. Murio, Automatic numerical differentiation by discrete mollification. Comput. Math. Appl. 13 (1987) 381-386.

[156] D.A. Murio, The mollification method and the numerical solution of ill-posed problems. John Wiley 1993.

[157] D.A. Murio \& L. Guo, Discrete stability analysis of the mollification method for numerical differentiation, Errata. Comput. Math. Appl. 19 (1990) 15-26, Ibidem 20 (1990) 75.

[158] D.A. Murio \& C.E. Mejia \& S. Zhan, Discrete mollification and automatic numerical differentiation. Comput. Math. Appl. 35 (1998) 1-16.

[159] M.Z. Nashed, On nonlinear ill-posed problems I: Classes of operator equations and minimization of functional. Pages 351-373 in Nonlinear analysis and applications (V. Lakshmikantham editor), Dekker,1987.

[160] F. Natterer, The finite element method for ill-posed problems. RAIRO Anal. Numer. 11 (1977) 271-278.

[161] F. Natterer, Numerical treatment of ill-posed problems. Pages 142-167 in Inverse Problems (G. Talenti editor), Lecture Notes in Mathematics 1225, Springer 1986.

[162] J. Oliver, An algorithm for numerical differentiation of a function of one real variable. J. Comput. Appl. Math. 6 (1980) 145-160.

[163] L.E. Payne, Bounds in the Cauchy problem for the Laplace equation. Arch. Rational Mech. Anal. 5 (1960) 35-45.

[164] L.E. Payne, On some non well-posed problems for partial differential equations. Pages 239-263 in Numerical solution of nonlinear differential equations ( Math. Res. Center Conference, Univ. of Wisconsin), Wiley 1966. 
[165] L.E. Payne, On a priori bounds in the Cauchy problem for elliptic equations SIAM J. Math. Anal. 1 (1970) 82-89.

[166] L.E. Payne, Some general remarks on improperly posed problems for partia differential equations. Pages 1-30 in Symposium on non-well-posed problem and logarithmic convexity, Lecture Notes in Math. 316, Springer, 1973.

[167] L.E. Payne, Improperly posed problems in partial differential equations. Re gional Conference Series in Applied Math. no.22, SIAM, 1975.

[168] L.E. Payne, On the stabilization of ill-posed Cauchy problems in nonlinea elasticity. Pages 1-10 in Problems of elastic stability and vibrations (Pitts burgh,1981), Contemp. Math. 4, Amer. Math. Soc., 1981.

[169] L.E. Payne, Improved stability estimates for classes of ill-posed Cauchy prob lems. Applicable Anal. 19 (1985) 63-74.

[170] L.E. Payne, On stabilizing ill-posed Cauchy problems for the Navier-Stoke equations. Pages 261-271 in Differential equations with applications to math ematical physics, Math. Sci. Engineering 192, Academic Press, 1993.

[171] L.E. Payne \& D. Sather, On some non-well-posed Cauchy problems for quasi linear equations of mixed type. Trans. Amer. Math. Soc. 128 (1967) 135-141

[172] L.E. Payne \& D. Sather, On an initial-boundary value problem for a class c degenerate elliptic operators. Ann. Mat. Pura Appl. 78 (1968) 323-338.

[173] Pham Minh Hien, A stable marching difference scheme for an ill-pose Cauchy problem for the three-dimensional Laplace equation. Vietnam J Math. 30 (2002) 79-88.

[174] P. Poláčik \& V. Šverák, Zeros of complex caloric functions and singularities c complex viscous Burgers equation. ArXiv:math.AP/0612506v1 18 Dec 2006

[175] A.P. Prudnikov \& Yu.A. Brychkov \& O.I. Marichev, Integrals and series Vol. 1: Elementary Functions. New York, Gordon \& Breach, 1986.

[176] C. Pucci, Studio col metodo delle differenze di un problema di Cauchy re] ativo ad equazioni alle derivate parziali del second'ordine di tipo parabolic (in Italian). Ann. Scuola Norm. Sup. Pisa 7 (1953) 205-215.

[177] C. Pucci, Sui problem di Cauchy non ben posti (in Italian). Atti Accad. Naz Lincei Rend. Cl. Sci. Fis. Mat. Natur. 18 (1955) 473-477.

[178] C. Pucci, Discussione del problema di Cauchy per le equazioni di tipo ellittic (in Italian). Ann. Mat. Pura Appl. (4) 46 (1958) 131-154.

[179] C. Pucci, Alcune limitazioni per le soluzioni di equazioni di tipo parabolic (in Italian). Ann. Mat. Pura Appl. 48 (1959) 161-172.

[180] A.G. Ramm, Numerical differentiation. Izv. Vyss. Ucebn. Zaved. Matematik 11 (1968) 131-134.

[181] A.G. Ramm, On stable numerical differentiation. Aust. J. Math. Anal. App] 5 (2008). 


\section{R. Magnanini and G. Talenti}

[182] A.G. Ramm \& A.B. Smirnova, On stable numerical differentiation. Math. Comp. 70 (2001) 1131-1153.

[183] Z. Ranjbar \& L. Eldén, Numerical analysis of an ill-posed Cauchy problem for a convection-diffusion equation. Inverse Probl. Sci. Eng. 15 (2007) 191-211.

[184] J. Rauch, Lectures on geometric optics. Pages 385-466 in Hyperbolic equations and Frequency Interactionsn (L. Caffarelli \& W. E Editors). Amer. Math. Soc., 1999.

[185] H.J. Reinhardt \& H. Han \& D.N. Hao, Stability and regularization of a discrete approximation to the Cauchy problem for Laplace equation. SIAM J. Numer. Anal. 36 (1999) 890-905.

[186] H.J. Reinhardt \& F. Seiffarth, On the approximate solution of ill-posed Cauchy problems for parabolic differential equations. Pages 284-298 in Inverse problems: principles and applications in geophysics, technology, and medicine (Potsdam, 1993), Math. Res. 74, Akademie-Verlag, 1993.

[187] L. Reichel, Numerical methods for analytic continuation and mesh generation. Constr. Approx. 2 (1986) 23-39.

[188] J. Rice \& M. Rosenblatt, Smoothing splines: regression, derivatives and disconvolution. Ann. Statist. 11 (1983) 141-156.

[189] R.D. Richtmyer, Principles of advanced mathematical physics, vol. 1. Springer, 1978.

[190] R.T. Rockafellar, Convex Analysis. Princeton Univ. Press, 1970.

[191] V.G. Romanov, A stability estimate for the solution to the ill-posed Cauchy problem for elasticity equations. J. Inverse Ill-Posed Problems 16 (2008) 615623.

[192] I. Sabba Stefanescu, On the stable analytic continuation with a condition of uniform boundedness. J. Math. Phys. 27 (1986) 2657-2686.

[193] E.I. Sakalauskas, The Galerkin-Tikhonov method of regularization in the problem of numerical differentiation (in Russian). Zh. Vychisl. Mat. I Mat. Fiz. 11 (1984) 1742-1747.

[194] R. Saylor, Numerical elliptic continuation. SIAM J. Numer. Anal. 4 (1967) $575-581$.

[195] D. von Seggern, CRC Standard curves and surfaces. CRC Press, 1993.

[196] R.E. Showalter, The final value problem for evolution equations. J. Math. Anal. Appl. 47 (1974) 563-572.

[197] R.E. Showalter, Quasi-reversibility of first and second order parabolic equations. Pages 76-84 in Research Notes in Mathematics, no.1, Pitman, 1975.

[198] S. Smale \& D-X Zhou, Estimating the approximation error in learning theory. Anal. Appl. (Singap.) 1 (2003) 17-41. 
[199] S. Smale \& D-X Zhou, Shannon sampling and function reconstruction fror point values. Bull. Amer. Math. Soc. 41 (2004) 279-305.

[200] S. Smale \& D-X Zhou, Shannon sampling. II. Connections to learning theory Appl. Comput. Harmon. Anal. 19 (2005) 285-302.

[201] S. Smale \& D-X Zhou, Learning theory estimates via integral operators anı their approximations. Constr. Approx. 26 (2007), 153-172.

[202] S. Steinberg, Some unusual ill-posed Cauchy problems and their applications Pages 17-23 in Improperly posed boundary value problems (Conf. Univ. Ner Mexico, 1974), Res. Notes in Math. 1, Pitman, 1975.

[203] T. Strom \& J.N. Lyness, On numerical differentiation. BIT 15 (1975) 314 322 .

[204] N.S. Surova, An inverstigation of the problem of reconstructing a derivativ by using an optimal regularizing integral operator. Numer. Methods Pro gramming 1 (1977) 30-34.

[205] G. Talenti, Sui problemi mal posti (in Italian). Bollettino U.M.I. 15-A (1978 $1-29$.

[206] A.N. Tikhonov, On stability of inverse problems (in Russian). Dokl. Akad Nauk. SSSR 39 (1944) 195-198.

[207] A.N. Tikhonov, On the solution of ill-posed problems and the method o regularization (in Russian). Dokl. Akad. Nauk. SSSR 151 (1963) 501-504

[208] A.N. Tikhonov, On the regularization of ill-posed problems (in Russian) Dokl. Akad. Nauk. SSSR 153 (1963) 49-52.

[209] A.N. Tikhonov, Improperly posed problems of linear algebra and a stabl method for their solution (in Russian). Dokl. Akad. Nauk SSSR 163 (1965 591-594.

[210] A.N. Tikhonov, On methods of solving incorrect problems. Translated fron the Russian original in Amer. Math. Soc. Transl. (2) 70 (1968) 222-224.

[211] A.N. Tikhonov \& V.Ya. Arsenin, Solution of Ill-Posed Problems. Wiley, 1977

[212] A.N. Tikhonov \& A.V. Goncharsky \& V.V. Stepanov \& A.G. Yagola, Nu merical methods for the solution of ill-posed problems. Mathematics and it Applications 328, Kluwer, 1995.

[213] A.N. Tikhonov \& A.S. Leonov \& A.G. Yagola, Nonlinear ill-posed prob lems, vol. 1 and 2, Applied Mathematics and Mathematical Computatios 14, Chapman \& Hall, 1998.

[214] D.D. Trong \& N.H. Tuan, Stabilized quasi-reversibility method or a class o nonlinear ill-posed problems. Electron. J. Differential Equations 84 (2008 pp. 35-55. 


\section{R. Magnanini and G. Talenti}

[215] M.M. Uzakov, Stability in multidimensional problems of analytic continuation (in Russian). Questions of well-posedness and methods for the investigation of inverse problems, 129-141, Vychisl. Tsentr, Novosibirsk, 1986.

[216] V.V. Vasin, The stable evaluation of a derivative in space $C(-\infty, \infty)$ (in Russian). USSR Computational Math. and Math. Phys 13 (1973) 16-24.

[217] V. Vapnik, Structure of statistical learning theory. John Wiley 1996.

[218] V. Vapnik, Statistical learning theory. John Wiley 1998.

[219] V. Vapnik, The nature of statistical learning theory. Springer-Verlag 2000.

[220] V. Vapnik, Estimation of dependence based on empirical data. SpringerVerlag 2006.

[221] S. Vessella, A continuous dependence result in the analytic continuation problem. Forum Math. 11 (1999) 695-703.

[222] N.A. Vrobeva \& L.S. Frank \& L.A. udov, Difference methods of solution of an ill-posed Cauchy problem for the three-dimensional Laplace equation (in Russian). Pages 147-155 in Computing methods and programming 11: numerical methods in gas dynamics, Izdat. Moskow Univ., 1968.

[223] Vu Kim Tuan, Stable analytic continuation using hypergeometric summation. Inverse Problems 16 (2000) 75-87.

[224] G.G. Walter, An alternative approach to ill-posed problems. J. Integral Equations Appl. 1 (1989) 287-301.

[225] J. Wang, Wavelet approach to numerical differentiation of noisy functions. Commun. Pure Appl. Anal. 6 (2007) 873-897.

[226] B.Ts. Zhamsoev, Estimates of stability in problems of analytic continuation (in Russian). Methods for solving inverse problems, 52-64, Vychisl. Tsentr, Novosibirsk, 1983.

[227] D. Zwillinger, Handbook of differential equations. Academic Press, 1984.

Rolando Magnanini and Giorgio Talenti

Dipartimento di Matematica "U. Dini"

Università di Firenze

viale Morgagni 67/A, 50134 Firenze

Italy

URL: http://www . math.unifi.it/users/magnanin

e-mail: magnanin@math.unifi.it

talenti@math.unifi.it

Lecture held by G. Talenti in the Seminario Matematico e Fisico on February 23, 2006

Received: May 5, 2009. 